\title{
Vortex Dynamics in Near Wake of a Hovering Hawkmoth
}

\author{
Hikaru Aono ${ }^{1}$ \\ Graduate School of Science and Technology, Chiba University, Chiba, 263-8522, Japan \\ Wei Shyy ${ }^{2}$ \\ Department of Aerospace Engineering, University of Michigan, Ann Arbor, MI, 48109, U.S.A. \\ and \\ Hao Liu $^{3}$ \\ Graduate School of Engineering, Chiba University, Chiba, 263-8522, Japan
}

\begin{abstract}
Numerical investigation of vortex dynamics in near wake of a hovering hawkmoth and hovering aerodynamics is conducted with a biology-inspired dynamic flight simulator. This simulator is developed to be capable of 'flying' an insect on a basis of realistic wing-body morphologies and kinematics. The computed results show a three-dimensional mechanism of vortical structures in hawkmoth hovering. A horseshoe-shaped vortex is observed, which wraps around each wing during the early down- and upstroke but subsequently grows into a doughnutshaped vortex ring, with an intense jet-flow present in its core, forming the downwash. The doughnut-shaped vortex rings of the wing pair eventually break up into two circular vortex rings downward in the wake. We furthermore evaluate the hovering energetics in terms of torques and powers based on the computed aerodynamic forces and velocity of the flapping wings.
\end{abstract}

\section{Nomenclature}

$\mathbf{a}_{0} \quad=$ acceleration (or deceleration) of the insect wing

$c_{\mathrm{m}} \quad=$ mean wing chord length (reference length)

$C_{x}, C_{y}, C_{z}=$ dimensionless force coefficients

$C_{D} \quad=$ dimensionless coefficient of horizontal (drag or thrust) force

$C_{L} \quad=$ dimensionless coefficient of vertical (lift) force

$C_{S} \quad=$ dimensionless coefficient of sideslip force

$d t \quad=$ time increment

$f \quad=$ flapping wing frequency

$\mathbf{F}_{\text {aero }} \quad=$ aerodynamic force

$\mathbf{F}_{\text {aero }} \quad=$ dimensionless aerodynamic force

$\mathbf{F}_{\text {aero, } i}^{*}=$ dimensionless aerodynamic force of the cell $(i)$

$i=$ cell index

$K=2 \pi f f\left(2 U_{\text {ref }}\right)=$ reduced frequency

$M_{m} \quad=$ mass of flight muscle

n $=$ unit outward normal vector

$\mathrm{O}=$ origin of earth-fixed Cartesian coordinates

$\mathrm{O}^{\prime}=$ origin of wingbase-fixed Cartesian coordinates

$p_{*} \quad=$ pressure

$\mathrm{P}^{*}{ }_{\text {aero }} \quad=$ dimensionless aerodynamic power

\footnotetext{
${ }^{1}$ Graduate student, hikaru@graduate.chiba-u.jp.

${ }^{2}$ Clarence L. "Kelly" Johnson Collegiate Professor and Chair, weishyy@umich.edu, Fellow AIAA

${ }^{3}$ Professor, hliu@faculty.chiba-u.jp
} 


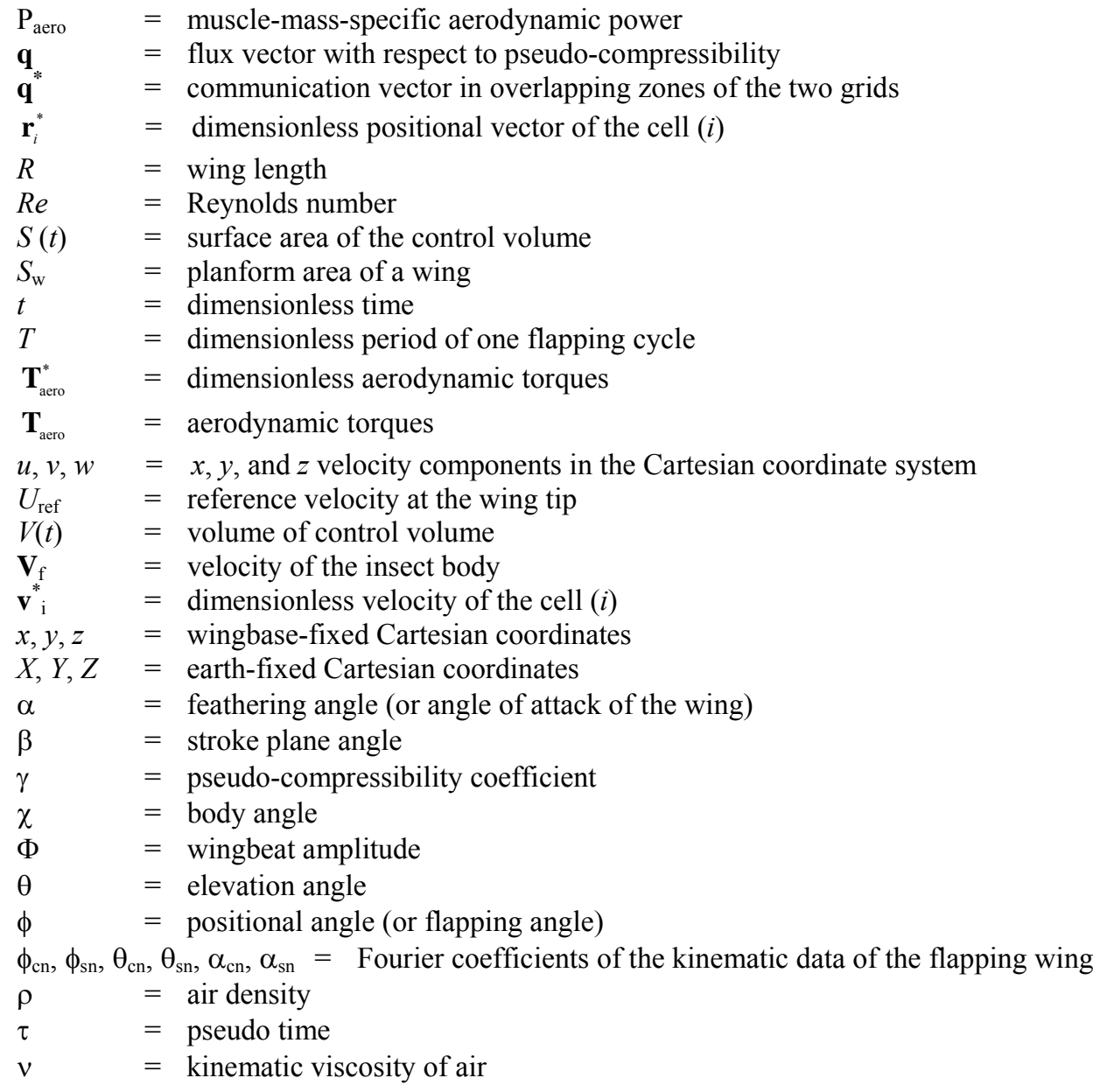

\section{Introduction}

Wake structure of insects in flapping flight is a key in understanding of the aerodynamics of insect flapping flight. ${ }^{1}$ It presents a footprint of the time-varying aerodynamics of flapping insects in terms of vortex dynamics and wake topology.

To quantify the vortex dynamics in near wake of an insect in flapping flight, a large numbers of experimental studies based on observations and measurements have been carried out in the past two decades. For an investigation of the wake visualization, Brodsky ${ }^{2}$ firstly measured the structure of the vortex wake for a peacock butterfly flying in a wind tunnel using high-speed filming. His results showed that the near wake of the butterfly in feeding flight was a system of discrete pairs of vortex rings and the dynamics of the vortex rings as well. Grodnitsky and Morozov $^{3,4}$ investigated near wake structures of several insects in tethered flight with a dust flow visualization technique. Their results indicated that flapping insects created a single vortex ring during each stroke and suggested that insects possessed special mechanisms for extracting energy back from the near vortex. Thereafter, with a smoke visualization technique that can provide flow information with higher resolution than the dust flow visualization, Ellington and his co-workers ${ }^{5-8}$ studied aerodynamics of hawkmoth flapping flight by investigating the near field flows around a real hawkmoth in tethered flight and by visualizing the wake and leading-edge vortex (LEV) around a robotic hawkmoth model wing in hovering flight. Dickinson and his co-workers ${ }^{9}$, by means of the PIV (particle imaging velocimetry) techniques, measured the near field flows around and aerodynamic forces acting on a robotic fly model wing in an idealized hovering motion. These studies have therefore revealed that insects utilize unsteady aerodynamic mechanisms to produce enough lift force to stay on aloft. In particular, prolonged attachment of a LEV has been recognized to be a key in enhancing the lift force production in insect flight. A transient LEV could be formed by sudden change in flow velocity or wing motion, and aerodynamic experiments in unsteady flows have shown that at its peak, the vortex can increase the lift coefficient markedly above the value which estimated by the steady-state theory. ${ }^{5-12}$ Recently, Thomas et al. ${ }^{13}$ and Bomphrey et al. ${ }^{14,}{ }^{15}$ investigated the LEV structure of the 
several real insects in forward flights with a high resolution using smoke-wire visualization and DPIV (digital particle image velocimetry) techniques. Their results indicated that the LEV could be continuous across the thorax, which could contribute aerodynamic force generation in a form of vortex-body interactions. More recently, Poelma et al. ${ }^{16}$ carried out a quantitative measurement of time-dependent three-dimensional velocity fields around a flapping wing.

The above-mentioned studies ${ }^{1-16}$ have therefore provided many quantitative features of the vortex dynamics in near wake and hence have deepened our understanding of the aerodynamics of insect flapping flight. However, the spatial and temporal resolutions in most of the experimental studies are not good enough to provide a clarified threedimensional mechanism of the vortical structures and its correlation with the aerodynamic force production. Compared with difficulties in experimental studies, CFD (computational fluid dynamic)-based studies may be an effective means. In fact, in the last decade CFD as a very useful tool has been widely applied in tackling the problem of flapping flight mechanisms. ${ }^{17-27}$ These simulation-based studies have shown great potential in dealing with the specific issues under consideration. However, most studies were either two-dimensional (2D) computations, which cannot represent the complex $3 \mathrm{D}$ vortex flow structures featured in insect flight; or they focused on a single wing and thereby neglected the effects of wing-wing and wing-body interactions.

In the present study, we investigate and address the vortex dynamics of unsteady 3D near wake in a hovering hawkmoth and its correlation with the aerodynamics force production by means of a biology-inspired dynamic flight simulator. $^{28,29}$ To reproduce a realistic hovering hawkmoth on a computer, both wing-body morphological and kinematic models are constructed based faithfully on the measurements of a real hawkmoth, Agrius convolvuli. Our computed results thereby quantify the vortex dynamics of near wake in a hovering hawkmoth in terms of the formation, development and shedding of vortices around the flapping wings and body. The simulations further integrate the vortex dynamics in the near field and wake topology in the far field as well as their correlations with the force generation and the effects of wing-wing and wing-body interactions.

\section{Methods and Materials}

We have developed an in-house computational system for quantifying the aerodynamics of a flapping-flying insect, named as 'a biology-inspired dynamic flight simulator'. ${ }^{28,29}$ This simulator is capable of 'flying' an insect with realistic wing-body morphologies and flapping-wing- and body-kinematics, and of evaluating unsteady aerodynamics including detailed vortex flow fields and flying energetics involving aerodynamic and inertial torques and powers. In the following, we give a brief description of the methodology of the simulator with a specific focus on three sub models: 1) a morphological model, 2) a kinematic model, and 3) a multi-block- and overset-grid based computational fluid dynamic (CFD) model.

\section{Morphological modeling}

We build a morphological model of an object (a wing-body insect model) in four-fold. First, we digitize the image of the object; secondly, we segment the image to extract the objects shape as a wire frame and/or skeleton model; thirdly, we make smoothing for the curves / surfaces of the wire frame to construct a 3D geometric model; and finally, we render the surface and/or volume to reconstruct the object and decompose the object in the computational domain to generate a grid. We further developed an efficient computer-aided method that unified morphological modeling and kinematic modeling of 3D flyers. ${ }^{26,30}$ With the modeling method, we constructed a realistic wing-body morphological model of the hawkmoth, Agrius convolvuli: the body length is $5.0 \mathrm{~cm}$ and the wing length is $5.05 \mathrm{~cm}$ (Fig. 1). Note that although the hawkmoth is four-winged, in the present study, they are modeled as a pair of wing considering that movement of fore-and-hind wing is nearly analogous ${ }^{31}$. We assumed a uniform wing thickness (1.2\% of the mean chord length) for the two wings, which resembles the wing geometry of a real insect. Note that to avoid the attachment of the wing on the body surface we added a virtual portion of the wing length (approximately $\mathrm{c}_{\mathrm{m}} / 32$, where $\mathrm{c}_{\mathrm{m}}$ is the mean wing chord length) at the wing base, which could largely improve the numerical convergence but seldom affect the results in the hovering flight.

\section{Kinematic modeling}

The kinematics of a flying insect comprises wingbeat and body movements (Fig. 2). The body movements are quantified by the body angle $\chi$ (inclination of the body relative to horizontal plane), and the stroke plane angle $\beta$ (plane in which the wing flaps). The wingbase-fixed coordinate system illustrated in Fig. 2A has its origin at the wing base, with the $x$-axis normal to the stroke plane, the $y$-axis perpendicular to the body axis, and the $z$-axis parallel to the stroke plane. The wingbeat movements are described by three positional angles within the stroke 
plane: 1) flapping about the $x$-axis in the wingbase-fixed coordinate system, described by the positional angle $\varphi ; 2$ ) rotation of the wing about the $z$-axis, described by the elevation angle $\theta$; and 3 ) rotation (feathering) of the wing about the $y$-axis by varying the angle of attack $\alpha$. Here, a general definition of the positional angle, the elevation angle and the angle of attack (feathering angle) are given in degree using the first three Fourier terms (Fig. 2B):

$$
\begin{aligned}
& \varphi(t)=\sum_{n=0}^{3}\left[\varphi_{\mathrm{cn}} \cos (n K t)+\varphi_{\mathrm{sn}} \sin (n K t)\right], \\
& \theta(t)=\sum_{n=0}^{3}\left[\theta_{\mathrm{cn}} \cos (n K t)+\theta_{\mathrm{sn}} \sin (n K t)\right], \\
& \alpha(t)=\sum_{n=0}^{3}\left[\alpha_{\mathrm{cn}} \cos (n K t)+\alpha_{\mathrm{sn}} \sin (n K t)\right] .
\end{aligned}
$$

Note that $t$ is dimensionless time and parameter $K$ is the reduced frequency defined by $2 \pi f c_{\mathrm{m}} / 2 U_{\text {ref }}$, where $f$ is flapping wing frequency, $c_{\mathrm{m}}$ is the mean wing chord length (reference length) and $U_{\text {ref }}$ is the reference velocity at the wing tip defined by $2 \Phi R f$, where $\Phi$ is the wingbeat amplitude and $R$ is the wing length. The Fourier coefficients $\varphi_{\mathrm{cn}}$, $\varphi_{\mathrm{sn}}, \theta_{\mathrm{cn}}, \theta_{\mathrm{sn}}, \alpha_{\mathrm{cn}}$ and $\alpha_{\mathrm{sn}}$ are determined accordingly where $n$ is integer varying from 0 to 3 . Reynolds number is defined as, $R e=c_{\mathrm{m}} U_{\text {ref }} / \mathrm{v}$, where $v$ is the kinematic viscosity of air, $1.5 \times 10^{-5} \mathrm{~m}^{2} \mathrm{~s}^{-1}$. Based on the measured data $\left(c_{\mathrm{m}}=\right.$ $1.83 \mathrm{~cm}, R=5.05 \mathrm{~cm}, \Phi=2.0 \mathrm{rad}(120$ degree $\left.), f=26.1 \mathrm{~s}^{-1}\right)$ of the hawkmoth ${ }^{31,32}, \operatorname{Re}$ and $K$ are calculated to be 6300 and 0.298 , respectively.

\section{Regridding for a flapping wing and a moving body}

The 3D movements of the flapping wing and the body cause large wing deformations and 6DOF (degree of freedom) displacements of the body. Modeling such movements requires an efficient and robust grid generator that fits the instantaneously deforming wing surface as well as the moved body and other boundaries. To model the 3D movements of a flapping wing (Fig. 2), we employ a previously described method $^{17,18,26}$ that uses the initial grid and the wing kinematics to analytically regenerate the wing-fitted grid, while minimizing additional computational requirements. The method is implemented in three-fold: 1) rotating grids in the whole wing-fitted sub-domain (the grid) according to the 'rigid' feathering motions of the wing; 2) rotating the feathering-based grids in the whole subdomain according to the 'rigid' flapping motion; and 3) rotating the feathering- and flapping-based grids in the whole sub-domain according to the elevation motion.

\section{Multi-block- and overset-grid method}

Modeling the shape of an insect with two or four wings is a challenging problem for CFD simulations. Not only are the wings undergoing large-scale movements relative to the body. They also flap rapidly, requiring us to model highly unsteady vortical flows about multiple and moving bodies. To achieve this, we develop a multi-blocked, overset grid method and incorporated into an in-house CFD solver. ${ }^{26,28-30,32}$ This grid method uses three individual structured grid system, one for the body and the one for each wing. Each grid system is made to fit the object (body or wing), moving and deforming with the object. A tri-linear interpolation technique ensures the communication of velocities and pressures among overlapping grids. ${ }^{26,28-30}$

As shown in Fig. 1B, three grids are generated for the body and the two wings of the hawkmoth. The wing grid comprises $45 \times 45 \times 31$ cells with the outer boundary 2 mean chord lengths away from the wing surface; and the grid for both wings are identical copies, using the relation of geometrical symmetry of the two wings about the body-axis. The body grid is much larger because it is used as a background grid to envelope the two wing grids for the interpolation; and it comprises $45 \times 47 \times 95$ cells, and the grid is approximately 20 mean chord lengths wide (measured as distance between outer boundary and body surface).

\section{Solutions to the Navier-Stokes equations}

The governing equations are the three-dimensional, incompressible unsteady Navier-Stokes (NS) equations, written in a strong conservative form for momentum and mass, and non-dimensionalized in an integral form, such that:

$$
\int_{V(t)}\left(\frac{\partial \mathbf{q}}{\partial \tau}\right) d V+\frac{\partial}{\partial t} \int_{V(t)} \mathbf{Q} d V+\int_{S(t)}\left(\mathbf{f}-\mathbf{Q} \mathbf{u}_{\mathrm{g}}\right) \cdot \mathbf{n} d S=0 .
$$


Where the term $\mathbf{f}=\left(\mathbf{F}+\mathbf{F}_{\mathrm{v}}, \mathbf{G}+\mathbf{G}_{\mathrm{v}}, \mathbf{H}+\mathbf{H}_{\mathrm{v}}\right)$ represents the net flux across the cell surfaces. The sub-terms are defined as:

$$
\begin{aligned}
& \mathbf{Q}=\left[\begin{array}{c}
u \\
v \\
w \\
0
\end{array}\right], \mathbf{q}=\left[\begin{array}{c}
u \\
v \\
w \\
p
\end{array}\right], \mathbf{F}=\left[\begin{array}{c}
u^{2}+p \\
u v \\
u w \\
z u
\end{array}\right], \mathbf{G}=\left[\begin{array}{c}
v u \\
v^{2}+p \\
v w \\
w
\end{array}\right] \\
& \mathbf{H}=\left[\begin{array}{c}
w u \\
w v \\
w^{2}+p \\
w w
\end{array}\right], \mathbf{F}_{\mathrm{v}}=-\frac{1}{\operatorname{Re}}\left[\begin{array}{c}
2 u_{x} \\
u_{y}+v_{x} \\
u_{z}+w_{x} \\
0
\end{array}\right], \mathbf{G}_{\mathrm{v}}=-\frac{1}{\operatorname{Re}}\left[\begin{array}{c}
v_{x}+u_{y} \\
2 v_{y} \\
v_{z}+w_{y} \\
0
\end{array}\right], \mathbf{H}_{\mathrm{v}}=-\frac{1}{\operatorname{Re}}\left[\begin{array}{c}
v_{z}+u_{x} \\
w_{y}+u_{z} \\
2 w_{z} \\
0
\end{array}\right]
\end{aligned}
$$

In the preceding equations, $\gamma$ is the pseudo-compressibility coefficient; $p$ is pressure; $u, v$, and $w$ are the $x, y$, and $z$ velocity components in the Cartesian coordinate system; $t$ denotes physical time, $\tau$ is pseudo time; $R e$ is the Reynolds number. Note that the term $\mathbf{q}$ associated with pseudo time is designed for an inner-iteration at each physical time step, and will vanish when the divergence of velocity is driven to zero so as to satisfy the equation of continuity. Time-dependent solutions of the incompressible Navier-Stokes equations are formulated in an ALE (Arbitrary Lagrangian-Eulerian) manner with the FVM (Finite Volume Method) and are performed in a timemarching manner with a pseudo-compressibility method; we enforce conservation of mass and momentum in both time and space. More details can be found in Liu. ${ }^{26}$

\section{Boundary conditions}

As shown in Fig. 1, the solutions to the NS equations with a multi-blocked, overset grid for a flapping insect require appropriate boundary conditions for the overlapping zones among the different single grid block, the moving walls of the wing and the body, and the far-field outside boundary.

For individual grid blocks and for the total wing and total body we use the fortified solutions to the NS equations by adding a forcing term with communication of a vector $\mathbf{q}^{*}$ to offer the boundary conditions for velocity and pressure in the overlapping zones of the two grids. The fortified equations are solved inside the computational domain, with except of holes and the single grid boundary. In the case of the hawkmoth, each time step requires that we solve the fortified NS equations three times, once for each grid cell block. On the body surface, the no-slip condition is applied to calculate the velocity components. To account for dynamic effects due to the accelerations of the oscillating body (moving and/or deforming body surface), pressure divergence at the surface stencils is derived from the local momentum equation, such that

$$
\begin{gathered}
(u, v, w)=\left(u_{\text {body }}, v_{\text {body }}, w_{\text {body }}\right) . \\
\partial p / \partial \mathbf{n}=-\mathbf{a}_{0} \cdot \mathbf{n} .
\end{gathered}
$$

Where the velocity $\left(u_{\text {body }}, v_{\text {body }}, w_{\text {body }}\right)$ and the acceleration $\left(\mathbf{a}_{0}\right)$ on the solid wall are evaluated and updated using the renewed grids on the body surface at each time step.

For the background grid of the insect body we need to define appropriate boundary conditions at the outside boundary (Fig. 1B). Consider that, when an insect hovers or flies forward at a speed $\mathbf{V}_{\mathrm{f}}$, the boundary conditions for the velocity and the pressure are given such as: 1$)$ at upstream $\mathbf{V}(u, v, w)=\mathbf{V}_{\mathrm{f}}$ while pressure $p$ is set to zero; 2$)$ at downstream zero-gradient condition is taken for both velocity and pressure, i.e., $\partial(u, v, w, p) / \partial \mathbf{n}=0$, where $\mathbf{n}$ is the unit outward normal vector at the outside boundary.

\section{Evaluation of forces, torques and powers}

The aerodynamic forces, $\mathbf{F}_{\text {aero }}^{*}$ of lift and drag coefficients acting on the wing can be calculated from the pressure and stresses along its surface based on the solutions to the NS equations. The resultant lift and thrust forces are calculated first in the local wingbase-fixed coordinate system $(x, y, z)$, and then transformed into the earth-based coordinate system $(X, Y, Z)$ accounting for the stroke plane angle (Fig. 2A), yielding vertical, horizontal and sideslip forces. Both the aerodynamic and inertial forces are non-dimensionalized with the reference velocity $U_{\text {ref, }}$, the reference length $c_{\mathrm{m}}$, the air density $\rho$, and the planform area of the wing $S_{\mathrm{w}}$, such that: 


$$
\mathbf{F}_{\text {aero }}^{*}=\frac{\mathbf{F}_{\text {aero }}}{0.5 \rho U_{\text {ref }}^{2} S_{\mathrm{w}}} .
$$

The dimensionless aerodynamic torque of the wing is calculated as the sum of the cross product of each force and the positional vector at each cell center of the wing about the origin of body (O'), such that:

$$
\mathbf{T}_{\text {aero }}^{*}=\sum_{i}\left(\mathbf{r}_{i}^{*} \times \mathbf{F}_{\text {aero }, i}^{*}\right) \text {. }
$$

Where $\mathbf{r}_{i}^{*}$ denotes the dimensionless positional vector of the cell center on the wing surface, and $\mathbf{F}_{\text {aero, } i}^{*}$ represents the aerodynamic force at each cell center, respectively.

The aerodynamic power is calculated as the scalar products of the velocity and the aerodynamic of the wing, such that:

$$
P_{\text {aero }}^{*}=\sum_{i}\left(\mathbf{F}_{\text {aero }, i}^{*} \cdot \mathbf{v}_{i}^{*}\right) \text {. }
$$

Where $\mathbf{F}_{\text {aero, } i}$ denotes the aerodynamic force at each cell center and $\mathbf{v}_{i}^{*}$ is the computed wing velocity at the cell $i$. Furthermore, to compare with the experimental result ${ }^{28}$ the muscle-mass-specific aerodynamic power can be calculated as:

$$
P_{\text {aero }}=P_{\text {aero }}^{*} / M_{m} \text {. }
$$

Where the mass of flight muscle, $M_{\mathrm{m}}$ is assumed to contribute up to $23 \%$ of the total body mass $(1.6 \mathrm{~g}){ }^{33}$

\section{Verification and validation}

Verification and validation of the single grid NS solver have demonstrated in the previous study. ${ }^{17}$ Moreover, verification and validation for the present in-house NS solver have been conducted through an extensive study of unsteady flows past a single, rowing-feathering fin with a single BFC (body-fitted coordinate) grid $(81 \times 31 \times 31)$ and with a two-block grid consisting of a single grid (case1: $51 \times 31 \times 25$ and case2: $31 \times 25 \times 13$ ) fitted to the fin and a cubic background grid $(81 \times 31 \times 31)$, at a Reynolds number $R e$ of $1.597 \times 10^{4}$ and the reduced frequency $K$ of 3.0 (Liu and Kato ${ }^{34}$ see Fig. 5). The computed results very well match those of with the single grid; and the computed time course of three force coefficients $C_{x}, C_{y}$, and $C_{z}$ show reasonable agreement with the robotic experiments (Liu and Kato ${ }^{34}$ see Fig. 6).

Verification of the present integrative simulator is further performed with a specific focus on its self-consistency in terms of grid refinement and time step effect. The results of the grid refinement in terms of the time courses of lift and drag forces coefficients are shown in Fig. 3A. We test four grid systems: case 1 (wing grid: $45 \times 45 \times 31$, body grid: $33 \times 35 \times 35$ ), case 2 (wing grid: $45 \times 45 \times 31$, body grid: $45 \times 47 \times 65$ ), case 3 (wing grid: $45 \times 45 \times 31$, body grid: $45 \times 47 \times 95$ ) and case 4 (wing grid: $45 \times 45 \times 45$, body grid: $45 \times 47 \times 95$ ). The plotted in Fig. $3 \mathrm{~A}$ indicates that the grid system of case 2 can provide reasonably accurate solutions for the hawkmoth hovering. The results of the effect of time increment on the aerodynamic force generation are also shown in Fig. 3B. We test two time steps of 0.01 and 0.005 . Fig. 3B shows almost no difference between the two cases and hence a physical time step of 0.01 is used throughout the simulations.

Furthermore, to validate the hovering flight of the hawkmoth, the mean aerodynamic forces are quantified by the following equations (11)-(13) using the mean aerodynamic force coefficients.

$$
\begin{aligned}
\text { Vertical force (lift) } & =0.5 \rho U_{\mathrm{ret}}^{2} S_{\mathrm{w}} C_{L}, \\
\text { Horizontal force (drag and thrust }) & =0.5 \rho U_{\mathrm{ref}}^{2} S_{\mathrm{w}} C_{D}, \\
\text { Sideslip force } & =0.5 \rho U_{\mathrm{ref}}^{2} S_{\mathrm{w}} C_{S},
\end{aligned}
$$

where $\rho$ is the density of air is the density of the air $\left(1.21 \mathrm{kgm}^{-3}\right), U_{\text {ref }}$ is the reference velocity, $S_{\mathrm{w}}$ is the planform area of the wing as well as $C_{L}, C_{D}, C_{S}$ are the three dimensionless mean force coefficients of the vertical, horizontal and sideslip forces, respectively. With the values assigned to the parameters defined in CFD simulation $\left(U_{\text {ref }}=5.05\right.$ $\left.\mathrm{ms}^{-1}\right)$ and equations (11) - (13), the mean vertical force $\left(17.1 \times 10^{-3} \mathrm{~N}\right)$ is calculated to be comparable to the weight of the hawkmoth $\left(15.7 \times 10^{-3} \mathrm{~N}\right)$; the horizontal and sideslip forces computed are less than $3 \%$ of the vertical force. These force prediction agree extremely well with the situation expected for the hovering flight and therefore indirectly validate our simulations. 


\section{Results}

Vortex dynamics in near wake

The absolute iso-vorticity surfaces around the hovering hawkmoth are illustrated in Fig. 4 at nine typical moments over a flapping cycle (see Fig. 2B). Note that the color of the absolute iso-vorticity surfaces indicates the magnitude of normalized helicity density which defines as the projection of a fluid's spin vector in the direction of its momentum vector, being positive (red) if it points in the same direction and negative (blue) if it points in the opposite direction.

In the first half of the downstroke (see Fig. 2B (b)), Fig. 4A-1, a horseshoe-shaped vortex is observed, which wraps around each wing and comprises a leading-edge vortex (LEV), a wing tip vortex (TV), and a trailing-edge vortex (TEV). These vortices are similar to the results of flow visualization observed in an experimental study (DPIV) of an impulsively-started dynamically scaled flapping wing by Poelma et al. ${ }^{16}$ As the flapping wings start acceleration downward, the LEVs and the TEVs grow in size and expand towards the wing base gradually. Apparently the TEVs from the right and left wings meet at the rear body (Fig. 4A-2), interacting with each other and then forming a complex vortical structure. During the middle downstroke (see Fig. 2B (c)), the TEVs are shed from the two wings but keep attached on the body. Eventually the shed TEV and TV are observed to join together (Fig. 4A-3). Immediately after that the LEVs are observed to break down at the location approximately $70-80 \%$ of the wing length. The LEV, the TV and the shed TEV in toto form a doughnut-shaped vortex ring for one wing, and hence a pair of vortex rings for the wing-pair. Focusing on the structure of the doughnut-shaped vortex ring near the wing tip, we find a twisted vortical structure (rolled-up) behind each wing, which result from an interaction between the broken-down LEV and the shed TV (Fig. 4A-4). Moreover, a root vortex is detected for each wing, which is shed from the wing and then connects with the shed TEV. Afterward a small vortex ring is observed for each wing adjacent to the body (Fig. 4A-5).

In the second half of the downstroke (see Fig. 2B (d)), the TVs enlarge gradually, and eventually when the wings approach the end of the downstroke the LEVs and TVs weaken down and detach from the wings. While the doughnut-shaped vortex rings of the wing pair break up into two circular vortex rings, forming the far-field wake below the hawkmoth, two small vortex rings of the wing pair is observed to join the two circular vortex rings (Fig. 4A-6). During most of the downstroke, the doughnut-shaped vortex ring pair has an intense, downward jet-flow through the 'doughnut' hole, which forms the downstroke downwash.

In the first half of the supination (see Fig. 2B (e)), as the flapping wings slow down the attached vortices (the LEVs and TVs) are shed from the wings. At this time, a pair of downstroke stopping vortices is observed wrapping the two wings (Fig. 4B-1). Subsequently, when the flapping wings begin to rotate quickly about the $y$-axis in the wing spanwise, a pair of upstroke starting vortex is detected around the wing tip and the trailing-edge (Fig. 4B-2). In the second half of the supination (Fig. 2B (f)), the upstroke starting vortices grow the TEVs and TVs are generated when the flapping wings accelerate rapidly. The downstroke wakes of the two circular vortex rings are captured evidently by each wing (Fig. 4B-3).

In the first half of the upstroke (Fig. 2B (g)), when the flapping wings begin to accelerate upward, the TEVs and TVs are shed from the two wings. After that the LEVs and the TVs are generated, which together with the TEVs, form a horseshoe-shaped vortex pair wrapping each wing (Fig. 4C-1). Subsequently just as during the downstroke, the horseshoe-shaped vortex grows and form a doughnut-shaped vortex ring for each wing. Similarly a pair of root vortices is detected as that during the downstroke, but it subsequently joins the vortex ring as illustrated in Fig. 4C-2. In the second half of the upstroke (Fig. $2 \mathrm{~B}(\mathrm{~h})$ ), the doughnut-shaped vortex rings elongate and deform while maintaining its ring shape and having the upstroke root vortex connected to the doughnut-shaped vortex rings (Fig. 4C-3). During most of the upstroke, the upstroke downwash through the center of each vortex ring is observed very similar to that during the downstroke.

During the early pronation (Fig. 2B (i)), attachment points of the shed upstroke TV move slightly (approximately $10-20 \%$ of the wing length) from the wing tip to the wing base due to the wing movement (Fig. 4D-1). At almost the same time, the upstroke stopping vortices are observed wrapping each wing (Fig. 4D-1). As the wings begin to rotate quickly, the upstroke stopping vortices are shed from the trailing-edge and the downstroke starting vortices are detected at the leading-edge and the wing tip. Thereafter the upstroke doughnut-shaped vortex rings are shed downward, breaking up into two vortex wake rings; the root vortices are also shed, forming a single vortex ring under the body (Fig. 4D-2). During the late pronation (Fig. 2B (a)), the downstroke starting vortices are initially observed at the leading-edge and the wing tip (Fig. 4D-3). 
Evaluation of hovering energetics: forces, torques and powers

The time courses of the aerodynamic forces of the wings and body are calculated by Eqs. (11) - (13) and plotted in Fig. 5: (A) vertical (lift) force, (B) horizontal (drag or thrust) force and (C) sideslip force, respectively. Figure 5 (A) indicates that a large lift generation is achieved when the wings approach nearly the middle down- and upstroke. As illustrated in Fig. 5 (B), the drag force is produced mainly during the downstroke and the thrust force is generated during the upstroke. Figure 5 (C) shows that the distinct sideslip force is generated mostly during earlyand late-pronation and early supination. At the middle supination and pronation, the aerodynamic forces (lift, drag (or thrust) and sideslip force) are fairly produced.

According to Eq. (8), the three aerodynamic torques (rolling, yawing and pitching moments, see Fig.2A) acting on the two wings are calculated. The time courses of the aerodynamic torques are plotted in Fig. 6: (A) rolling, (B) pitching and (C) yawing, respectively. Note that the term of 'total wing' in Fig.6 indicates the total torque of the wings that is a sum of the torques of the right and left wings. Although three aerodynamic torques are relatively comparable, the aerodynamic rolling (ART) and yawing (AYT) torques of the two wings are much smaller than those of the aerodynamic pitching torque (APT). Furthermore, time-varying total ART and total AYT are mostly zero over a flapping cycle because of symmetrical flapping wing motion and therefore the mean total ART and total AYT are approximately zero (Fig. 6(A) and (C)). In addition, the APT of the two wings is produced by the aerodynamic forces, which vary over a wide range from $-0.6 \times 10^{-3} \mathrm{Nm}$ to $0.4 \times 10^{-3} \mathrm{Nm}$ during a flapping cycle. Note that a signature of the APT means that negative of the APT makes the body attitude of the insect tends to noseup and vice versa. The transient APT of the two wings peaks fourth, at early and late pronation and supination. In short, the prediction of the APT of the wings show that the body may experience a nose-up pitching torque during the late pronation and the first half of the downstroke, and a nose-down pitching torque during the late supination and the first half of the upstroke as well.

Based on the computed transient aerodynamic forces and the dynamic velocity of the flapping wings, the muscle-mass-specific aerodynamic $P_{\text {aero }}$ can be calculated by Eqs. (9) and (10). The $P_{\text {aero }}$ means the power necessary to overcome air resistance. The time courses of the $P_{\text {aero }}$ of the wings are plotted in Fig. 7 . The maximums of the $P_{\text {aero }}$ are predicted during both early down- and upstroke corresponding with the transient aerodynamic forces. In addition, the transient $P_{\text {aero }}$ maintains a large amplitude sweep during the down- and upstroke. The computed mean $P_{\text {aero }}\left(87.2 \mathrm{Wkg}^{-1}\right)$ is very close to the experimental results by Willmott and Ellington. ${ }^{33}$

\section{Conclusions}

We have presented a numerical investigation of the unsteady 3D near wake in hawkmoth hovering and the hovering energetics. To quantify detailed near- and far-field vertical structures around hovering hawkmoth, we built up a hawkmoth model which has realistic wing-body morphologies and can mimic 3D flapping-wing kinematics. The simulation was validated to be able to provide highly resolved unsteady aerodynamics in insect hovering. Based on our computed results, the vortex dynamics in near wake of a hovering hawkmoth and aerodynamics of the hovering hawkmoth can be clarified as follows.

\section{Vortex dynamics of near wake around a hovering hawkmoth}

Our computed results show that a key feature of the near wake in a hawkmoth hovering is the generation of the doughnut-shaped vortex rings of the wing pair during most of the down- and upstroke as shown in Fig. 4. The doughnut-shaped vortex ring eventually detaches from the wing and body during the supination and pronation, subsequently breaking up into two circular vortex rings, with strong downward flow through the core (hole) of the vortex ring. These phenomena of a vortex ring and its breaking-up were also predicted by Van den Berg and Ellington $^{8}$ in an experimental study on the basis of an analysis of the vortex wake structures around a robotic hawkmoth model. Van den Berg and Ellington ${ }^{8}$ also observed a vortex wake ring (they called it dumbbell-shape vortex) with an intensive downwash through its centre in the wing wake during the downstroke. However, they did not quantify vortex dynamics which indicate the formation, development, and break-up of the vortex ring because of the technical limitations of their smoke-rake flow visualization. Especially, our computed results show the twisted vortical structure (rolled-up) around the wing tip due to an interaction between the broken-down LEVs and the TVs. Even though, they predicted that the dumbbell-shape vortex structure should eventually break up into two single circular vortex rings rather than merge together into a single one. Moreover the computed vortex wake structure during the downstroke of the hawkmoth analyzed here also differs from the descriptions of the shed vortex wake observed in previous studies of insect flight. ${ }^{3,4,6,9,10,35,36}$ Another interesting finding is that there is an interaction between the body and wake (the shed TEV) during the early downstroke. This flow phenomenon has not been reported by previous studies $3,4,6,9,10,35,36$ because of ignorance of the effect of the body on the flow field and 
insufficient visualized resolution in space. Therefore our computed results show that the vortex interaction upon the body plays a key role in the formation of near wake (doughnut-shaped vortex ring) of the hawkmoth in hovering flight (Fig.4).

\section{Aerodynamics of hawkmoth hovering}

Focusing on the aerodynamics force generation, we find two peaks of lift force during each stroke in hawkmoth hovering. Recalling the computed flows shown in Fig. 4, we believe that the first lift peak is responsible for the LEV lift-boot mechanism (delayed stall ${ }^{5,12}$ ), while the second lift peak is likely to be associated with rabbit vorticity increase as the wing experiences fast pitching-up rotation. Such a view point has also been held by other studies. ${ }^{5-12}$, $17-19,37$

With respect to the wing aerodynamic torques, we note that the computed aerodynamic yawing and rolling torques are canceled out due to the symmetric wing kinematics even though the aerodynamic pitching torque shows significant variation with time. Considering the effects of the body attitude in hovering flight, the time-varying APT could make a longitudinal oscillation over a flapping wing cycle. Similar longitudinal oscillation of the body has also reported by Wang et al.. ${ }^{38}$ This implies that evaluation of the time-varying APT could be a key issue in understanding flight control mechanisms in hovering insects.

The muscle-mass-specific aerodynamic power of the wings shows a wave form very similar to that of the aerodynamic forces. This may point to a fact that a hovering hawkmoth costs much aerodynamic power of the wings due to the large aerodynamic force production. Interestingly, during the supination and pronation, the magnitude of the aerodynamic force-based power requirement is much smaller than that of during the down- and upstroke. Therefore a hovering hawkmoth during the supination and pronation may not spend much power to practically move the wings in the air based on the aerodynamic force-based power estimation.

\section{Acknowledgments}

The present work was partially supported by a PRESTO (Precursory Research for Embryonic Science and Technology) program of the Japan Science and Technology Agency (JST), and the Grant-in-Aid for Scientific Research of No. 18656056 and No. 18100002, Japan Society for the promotion of Science (JSPS). The simulations were performed in a supercomputer (RSCC), RIKEN (The Institute of Physical and Chemical Research), Japan.

\section{References}

${ }^{1}$ Brodsky, A. K., The evolution of insect flight, Oxford University Press, Oxford, 1984, Chaps. 3.

${ }^{2}$ Brodsky, A. K., "Vortex Formation in the Tethered Flight of the Peacock Butterfly Inachis Io L. and Some Aspects of Insect Flight Evolution," Journal of Experimental Biology, Vol. 161, 1991, pp. 77-95.

${ }^{3}$ Grodnitsky, D. L. and Morozov, P. P., "Flow Visualization Experiments on Tethered Flying Green Lacewings Chrysopa Dasyptera," Journal of Experimental Biology, Vol.169, 1992, pp. 143-163.

${ }^{4}$ Grodnitsky, D. L. and Morozov, P. P., "Vortex Formation during Tethered Flight of Functionally and Morphologically Twowinged Insects, Including Evolutionary Considerations on Insect Flight,” Journal of Experimental Biology, Vol. 182, 1993, pp. $11-40$.

${ }^{5}$ Ellington, C. P., van den Berg, C., Willmott, A. P. and Thomas, A. L. R., "Leading-edge Vortices in Insect Flight," Nature, Vol. 384, 1996, pp. 626-630.

${ }^{6}$ Willmott, A. P., Ellington, C. P. and Thomas, A. L. R., "Flow Visualization and Unsteady Aerodynamics in the Flight of the Hawkmoth, Manduca sexta.," Philosophical Transactions of the Royal Society of London, Series B, Vol. 352, 1997, pp. $303-316$.

${ }^{7}$ Van den Berg, C. and Ellington, C. P., "The Three-dimensional Leading-edge Vortex of a 'Hovering' Model Hawkmoth," Philosophical Transactions of the Royal Society of London, Series B, Vol. 352, 1997, pp. 329-340.

${ }^{8}$ Van den Berg, C. and Ellington, C. P., "The Vortex Wake of a 'Hovering' Model Hawkmoth," Philosophical Transactions of the Royal Society of London, Series B, Vol. 352, 1997, pp. 317-328.

${ }^{9}$ Ellington, C. P., "The Aerodynamics of Insect Flight. IV. Aerodynamic Mechanisms," Philosophical Transactions of the Royal Society of London, Series B, Vol. 305, 1984, pp. 79-113.

${ }^{10}$ Ellington, C. P., "The Aerodynamics of Insect Flight. V. A Vortex Theory," Philosophical Transactions of the Royal Society of London, Series B, Vol. 305, pp. 115-144.

${ }^{11}$ Dickinson, M. H., Lehmann, F.-O. and Sane, S., "Wing Rotation and the Aerodynamic Basis of Insect Flight," Science, Vol. 284, 1999, pp. 1954-1960.

${ }^{12}$ Shyy, W. and Liu, H., "Flapping Wings and Aerodynamic Lift: the Role of Leading-edge Vortices", AIAA Journal, Vol. 45(2), 2007, pp. 2819-2821.

${ }^{13}$ Thomas, A. L. R., Bomphrey, R. J., Srygley, R. B., Nudds, R. L. and Taylor, G. K., "Dragonfly Flight: Free-flight and Tethered Flow Visualizations Reveal a Diverse Array of Unsteady Lift Generating Mechanisms, Controlled Primarily via Angle of Attack," Journal of Experimental Biology, Vol. 207, 2004, pp. 4299-4323. 
${ }^{14}$ Bomphrey, R. J., Lawson, N. J., Taylor, G. K. and Thomas, A. L. R., "The Aerodynamics of Manduca sexta: Digital Particle Image Velocimetry Analysis of the Leading-edge Vortex," Journal of Experimental Biology, Vol. 208, 2005, pp. 10791094.

${ }^{15}$ Bomphrey, R. J., Lawson, N. J., Taylor, G. K. and Thomas, A. L. R., "Application of Digital Particle Image Velocimetry to Insect Aerodynamics: Measurement of the Leading-edge Vortex and Near Wake of a Hawkmoth," Experiments in Fluids, Vol. 40, 2006, pp. 546-554.

${ }^{16}$ Poelma, C., Dickson, W. B. and Dickinson, M. H., "Time-resolved Reconstruction of the Full Velocity Field Around a Dynamically-scaled Flapping Wing," Experiments in Fluids, Vol. 41, 2006, pp. 213-225.

${ }^{17}$ Liu, H. and Kawachi, K., "A Numerical Study of Insect Flight,” Journal of Computational Physics, Vol. 146, 1998, pp. 124-156.

${ }^{18}$ Liu, H., Ellington, C. P., Kawachi, K., van den Berg, C., and Willmott, A. P., "A Computational Fluid Dynamic Study of Hawkmoth Hovering,” Journal of Experimental Biology, Vol. 201, 1998, pp. 461-477. 2219.

${ }^{19}$ Wang, Z. J., "Two Dimensional Mechanism for Insect Hovering,” Physical Review Letters, Vol. 85, No.10, 2000, pp. 2216-

${ }^{20}$ Ramamurti, R. and Sandberg, W. C., "A Three-dimensional Computational Study of the Aerodynamic Mechanisms of Insect Flight," Journal of Experimental Biology, Vol. 205, 2002, pp. 1507-1518.

${ }^{21}$ Ramamurti, R. and Sandberg, W. C., "A Computational Investigation of the Three-dimensional Unsteady Aerodynamics of Drosophila Hovering and Maneuvering," Journal of Experimental Biology, Vol. 210, 2007, pp. 881-896.

${ }^{22}$ Sun, M. and Tang, J., "Lift and Power Requirements of Hovering Flight in Drosophila virlis," Journal of Experimental Biology, Vol. 205, 2002, pp. 2413-2427.

${ }^{23}$ Wang, Z. J., Birch, J.M. and Dickinson, M. H., "Unsteady Forces and Flows in Low Reynolds Number Hovering Flight: Two-dimensional Computations vs Robotic Wing Experiments," Journal of Experimental Biology, Vol. 207, 2004, pp. 449-460.

${ }^{24}$ Miller, L. A. and Peskin, C. S., "When Vortices Stick: an Aerodynamic Transition in Tiny Insect Flight," Journal of Experimental Biology, Vol. 207, 2004, pp. 3073-3088.

${ }^{25}$ Miller, L. A. and Peskin, C. S., "A Computational Fluid Dynamics of 'Clap and Fling' in the Smallest Insects," Journal of Experimental Biology, Vol. 208, 2005, pp. 195-212.

${ }^{26}$ Liu, H., "Simulation-based Biological Fluid Dynamics in Animal Locomotion," ASME Applied Mechanics Reviews, Vol. 58, No. 4, 2005, pp. 269-282.

${ }^{27}$ Shyy, W., Lian, Y.S., Tang, J., Viieru, D. and Liu, H., Aerodynamics of Low Reynolds Number Flyers, Cambridge University Press, 2007.

${ }^{28}$ Aono, H., Fuyou, L. and Liu, H., "Near- and Far-field Aerodynamics of Insect Hovering Flight: an Integrated Computational Study" Journal of Experimental Biology, 2008 (in press).

${ }^{29} \mathrm{Liu}, \mathrm{H} .$, "Integrative Modeling of Insect Flight: Morphology, Kinematics and Aerodynamics," (in preparation).

${ }^{30}$ Liu, H., "Computational Biological Fluid Dynamics: Digitizing and Visualizing Animal Swimming and Flying," Integrative and Comparative Biology, Vol. 42, 2002, pp. 1050-1059.

${ }^{31}$ Willmott, A. P. and Ellington, C. P, "The Mechanics of Flight in the Hawkmoth, Manduca sexta I: Kinematics of Hovering and Forward Flight,” Journal of Experimental Biology, Vol. 200, 1997, pp. 2705-2722.

${ }^{32}$ Aono, H. and Liu, H., "Vortical Structure and Aerodynamics of Hawkmoth Hovering," Journal of Biomechanical Science and Engineering, Vol. 1, No. 1, 2006, pp. 234-245.

${ }^{33}$ Willmott, A. P. and Ellington, C. P, "The Mechanics of Flight in the Hawkmoth, Manduca sexta II: Aerodynamics Consequences of Kinematics and Morphological Variation," Journal of Experimental Biology, Vol. 200, 1997, pp. 2723-2745

${ }^{34}$ Liu, H. and Kato, N., "A Numerical Study of Unsteady Hydrodynamics of a Mechanical Pectoral Fin," Journal of Bionics Engineering, Vol. 1, No. 2, 2004, pp. 108-120.

${ }^{35}$ Birch, J. M. and Dickinson, M. H., "Spanwise Flow and the Attachment of the Leading-edge Vortex on Insect Wings, Nature, Vol. 412, 2001, pp. 729-733.

${ }^{36}$ Dickinson, M. H., and Götz, K. G., "The Wake Dynamics and Flight Forces of the Fruit Fly, Drosophila melanogaster," Journal of Experimental Biology, Vol. 199, 1996, pp. 2085-2104.

${ }^{37}$ Viieru, D., Tang, J., Lian, Y., Liu, H. and Shyy, W., "Flapping and Flexible Wing Aerodynamics of Low Reynolds Number Flight Vehicles," AIAA paper 2006-0503, presented at the 44th Aerospace Sciences Meeting and Exhibit, Reno, NV, Jan. 9-12, 2006.

${ }^{38}$ Wang, H., Inada, Y. and Liu, H., "A Numerical Analysis of Inertial Torques in the Steering Maneuvers of Hovering Drosophila," JSME International Journal, Series C, Vol. 48, No. 4, 2005, pp. 499-512.

${ }^{39}$ Ellington, C. P., "Aerodynamics of Insect Flight. III. The Kinematics," Philosophical Transactions of the Royal Society of London, Series B, Vol. 305, 1984, pp. 79-113. 
(A)

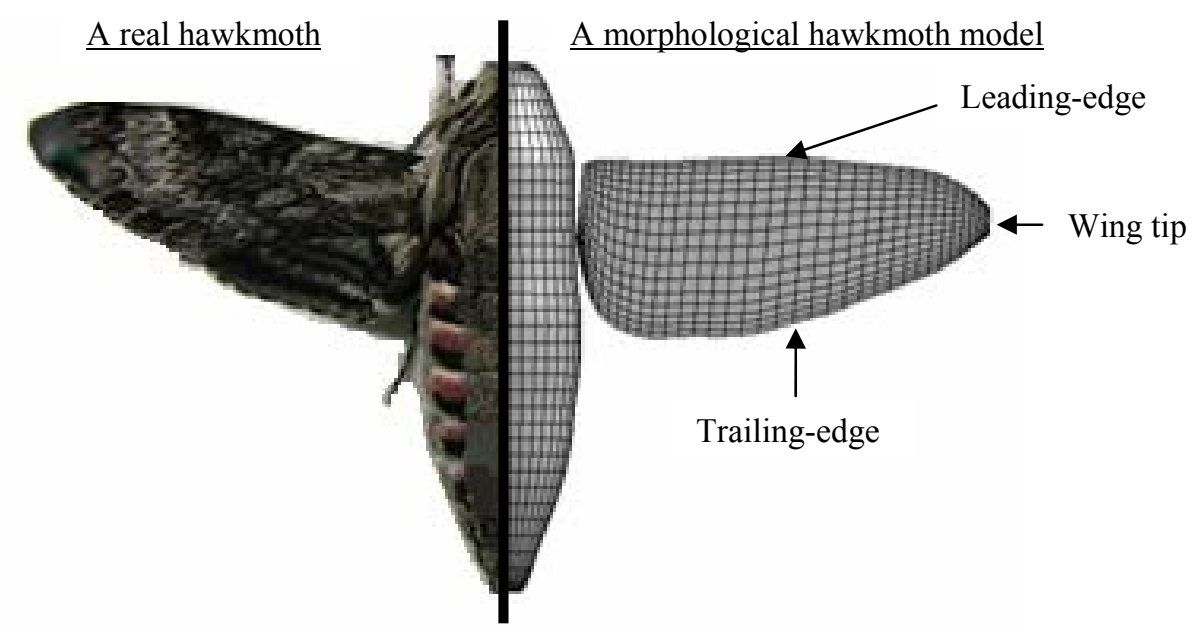

(B)

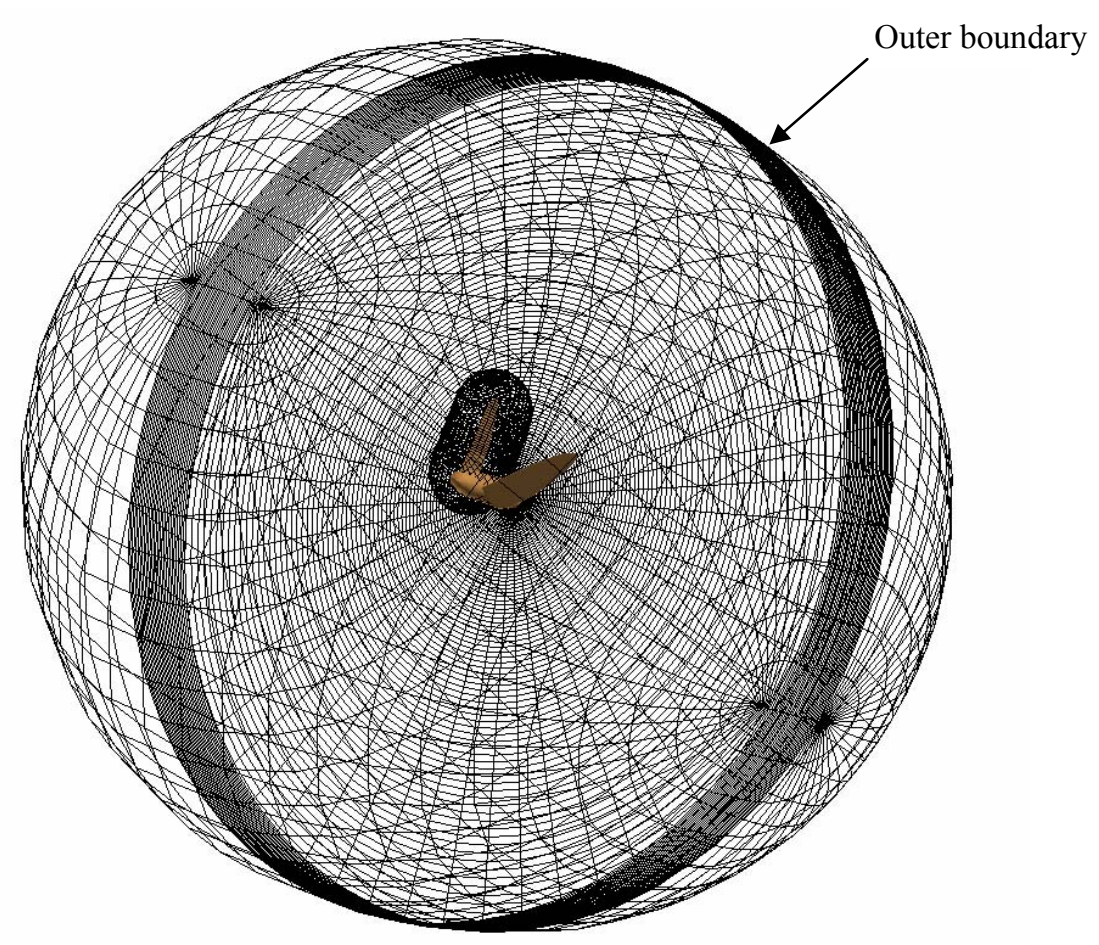

Figure 1 A morphological model of a hawkmoth, Agrius convolvuli.

(A) A hawkmoth, Agrius convolvuli with a computational model superimposed on the right half. The hawkmoth has a body length of $5.0 \mathrm{~cm}$, a wing length of $5.05 \mathrm{~cm}$ (mean wing chord length $c_{\mathrm{m}}=1.83 \mathrm{~cm}$ ), and an aspect ratio of 2.76

(B) A multi-block grid system of the two wings and body of the hawkmoth (wing: $45 \times 45 \times 31$, body: $45 \times 47 \times 95$ ) with a distance between the body surface and the outer boundary of $20 c_{\mathrm{m}}$. 
(A)
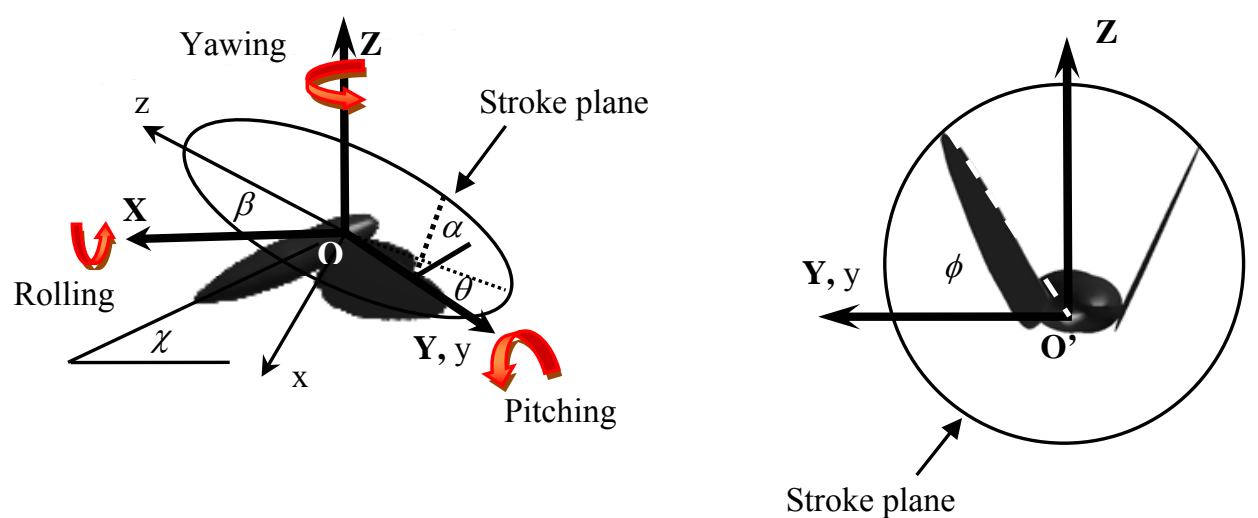

(B)

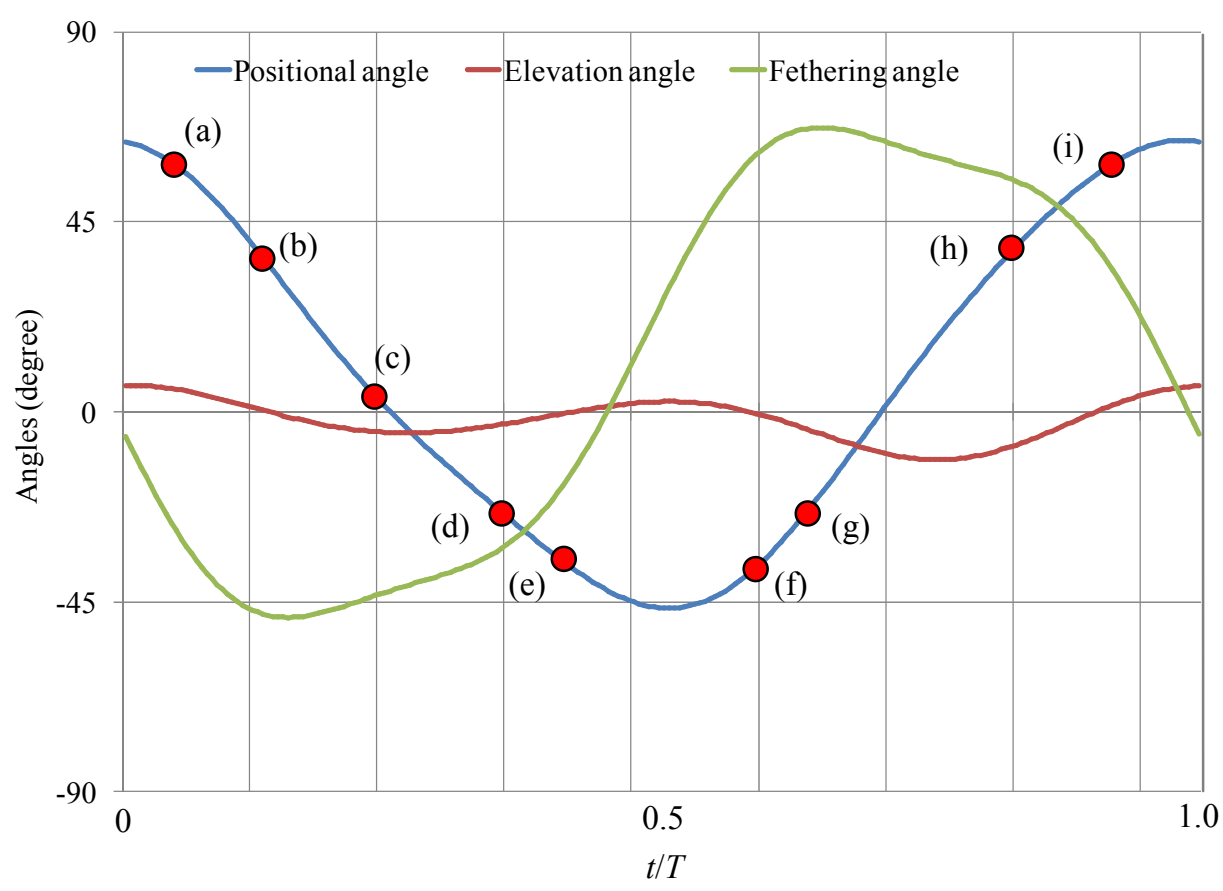

Figure 2 Schematic diagrams of the computational system of a hawkmoth, Agrius convolvuli.

(A) The local wingbase-fixed $(x, y, z)$ and the global earth-fixed $(X, Y, Z)$ coordinate systems. The origin $\mathrm{O}^{\prime}$ of the wingbase-fixed coordinate system lies at the wing base, with the $x$-axis normal to the stroke plane (the $y z$ plane as defined by Ellington ${ }^{39}$, the $y$-axis vertical to the body axis and $z$-direction parallel to the stroke plane. The wing kinematics are described by the positional angle $\varphi$, the feathering angle (angle of attack of the wing) $\alpha$, the elevation angle $\theta$, and the stroke plane angle $\beta$; the link to the earth-fixed frame of reference comes through the body angle $\chi$. We assume a body angle $\chi$ of $39.8^{\circ}$ and a stroke plane angle $\beta$ of $15.0^{\circ}$ (Willmott and Ellington ${ }^{33}$ ).

(B) Instantaneous positional angle $\varphi$, feathering angle $\alpha$, and elevation angle $\theta$ of the hawkmoth wing over one complete flapping cycle. Blue, dark red and green lines represent the positional angle $\varphi$, the feathering angle $\alpha$ and the elevation angle $\theta$, respectively. Red points marked: (a) late pronation, (b) early downstroke, (c) mid downstroke, (d) late downstroke, (e) early supination, (f) late supination, (g) early upstroke, (h) late upstroke and (i) early pronation. $T$ denotes dimensionless period of one flapping cycle. 
(A)

Lift force coefficients

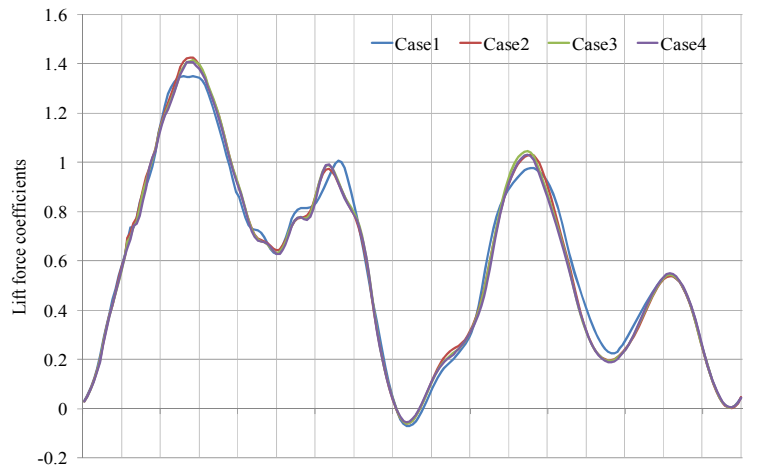

$(\alpha-1)$ Wing grid

Drag force coefficients

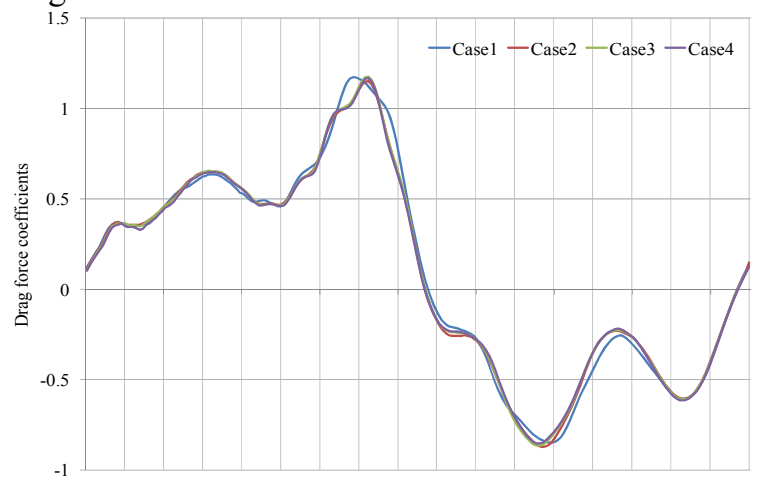

$(\alpha-2)$ Wing grid

(B)

Lift force coefficients

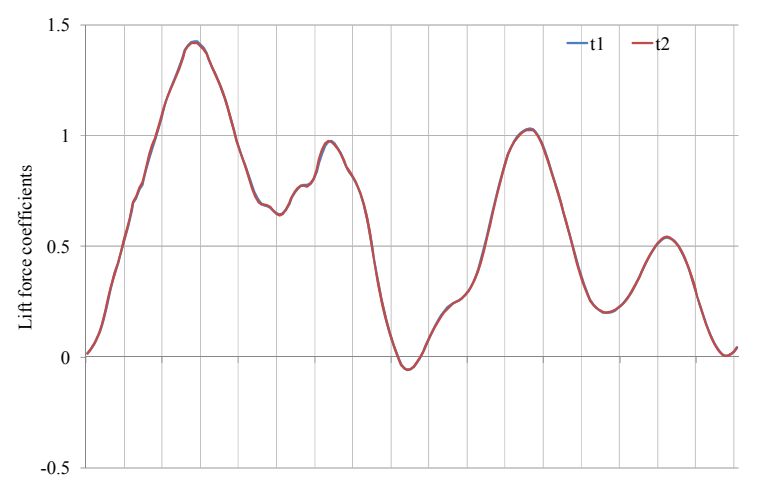

$(\alpha-3)$ Wing grid

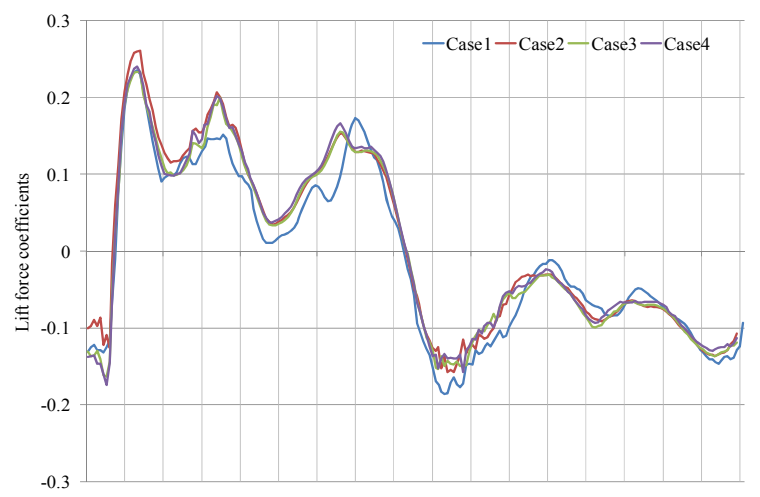

( $\beta-1)$ Body grid

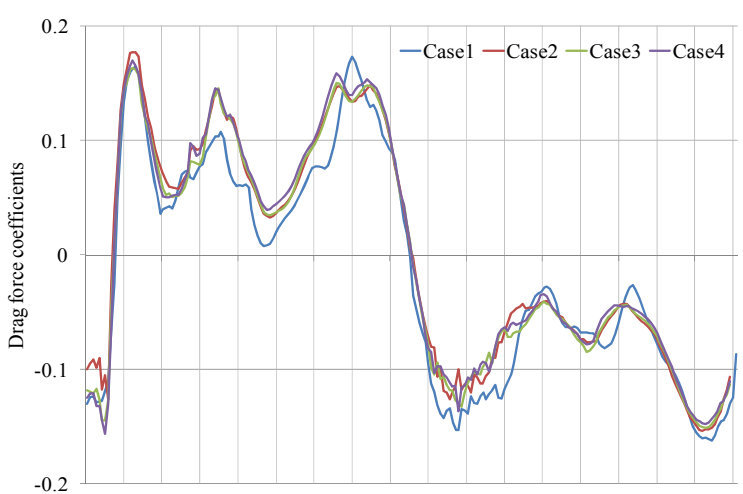

$(\beta-2)$ Body grid

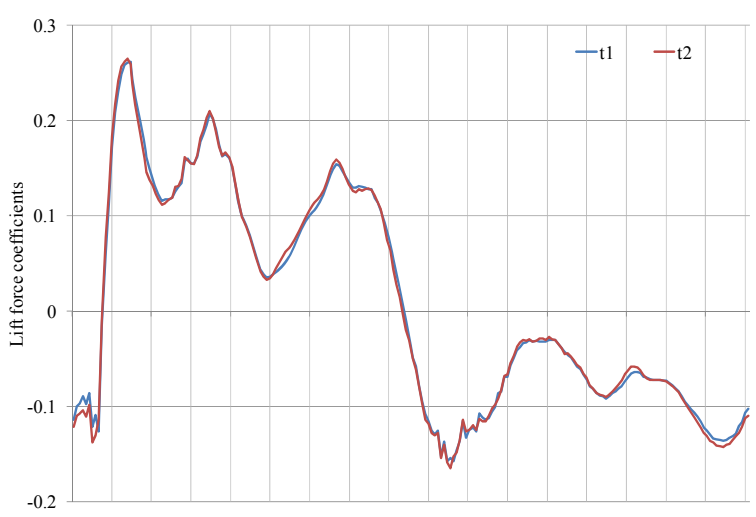

$(\beta-3)$ Body grid 


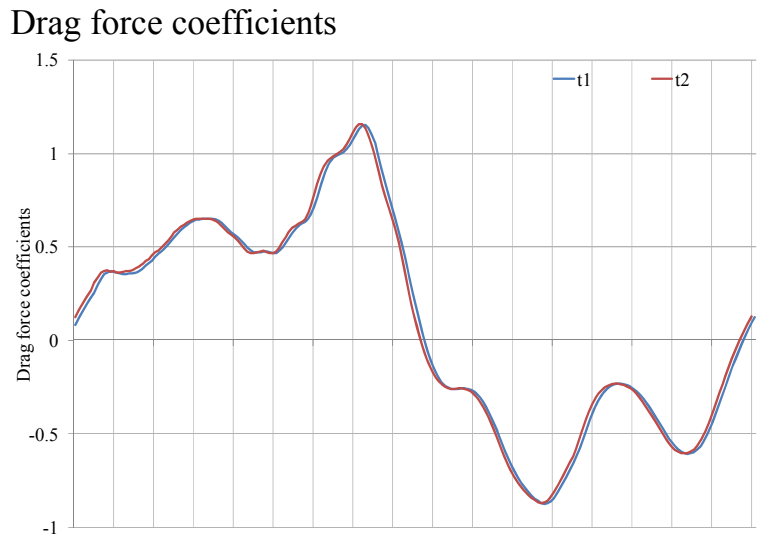

$(\alpha-4)$ Wing grid

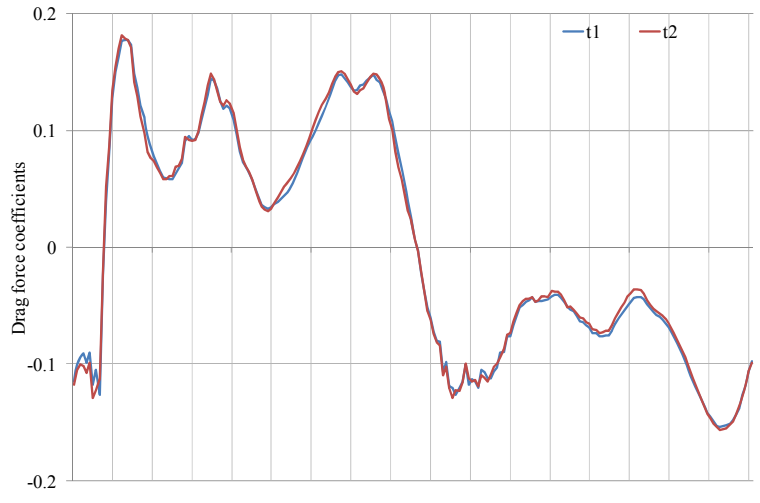

$(\beta-4)$ Body grid

Figure 3 Effects of grid and time step on aerodynamic force generation over a flapping cycle.

(A) Grid sensitive analysis for lift and drag force coefficients acting on a wing $(\alpha-1,2)$ and a body $(\beta-1,2)$, respectively. Four grid systems are used: case1 (wing grid: $45 \times 45 \times 31$, body grid: $33 \times 35 \times 35$ ), case 2 (wing grid: $45 \times 45 \times 31$, body grid: $45 \times 47 \times 65$ ), case 3 (wing grid: $45 \times 45 \times 31$, body grid: $45 \times 47 \times 95$ ) and case 4 (wing grid: $45 \times 45 \times 45$, body grid: $45 \times 47 \times 95$ ), respectively.

(B) Time step sensitive analysis for lift and drag force coefficients acting on a wing $(\alpha-3,4)$ and a body $(\beta-3,4)$, respectively. Two time steps are used: $\mathrm{t} 1$ (blue lines), a time step $d t$ of 0.01 and $\mathrm{t} 2$ (red lines), a time step $d t$ of 0.005 . 
A-1

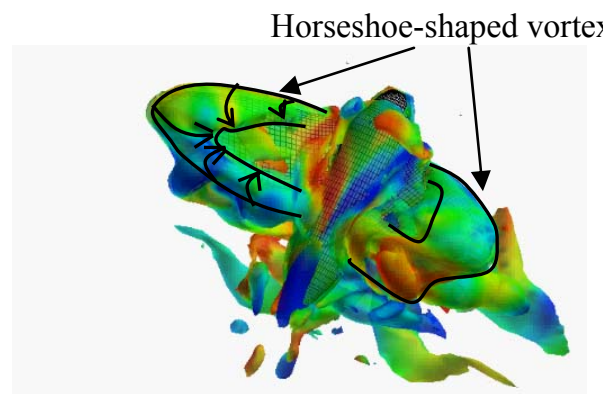

$\gamma_{r}$

A-3

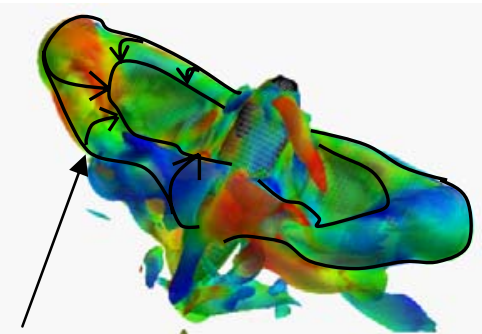

Joint of the shed TEV and the shed TV

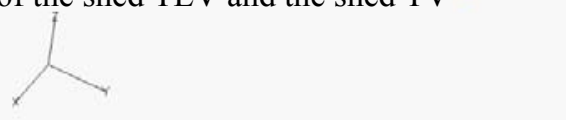

A-5

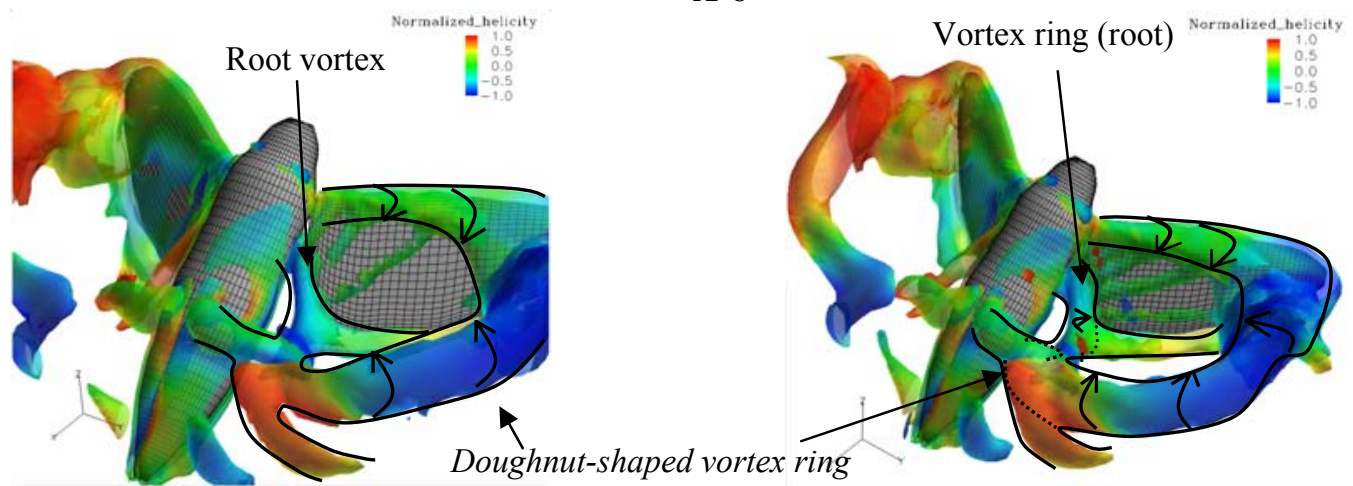

B-1

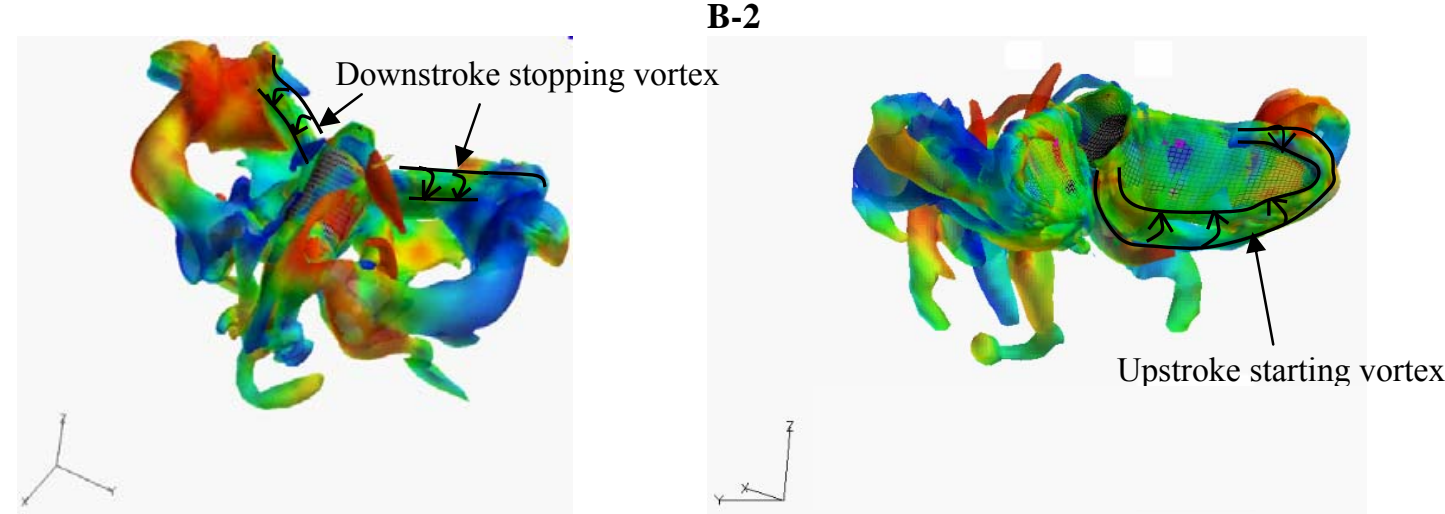

American Institute of Aeronautics and Astronautics 
B-3
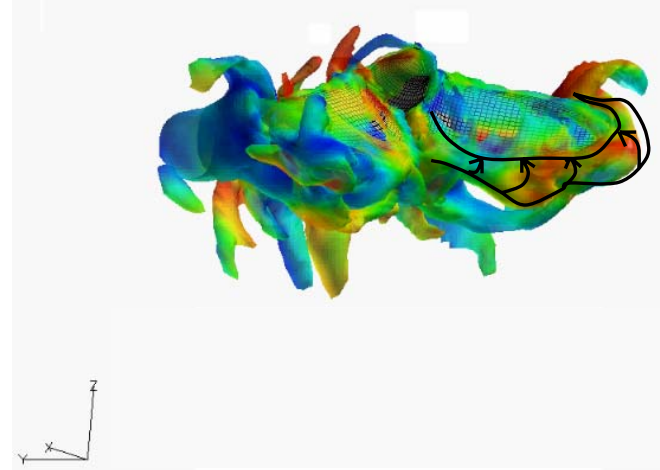

C-1
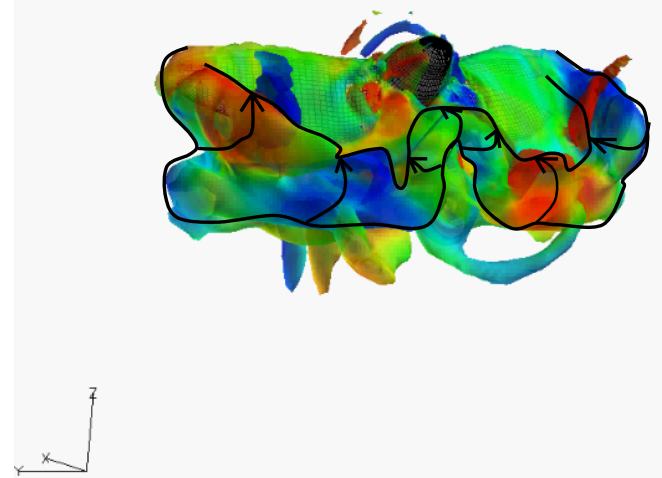

C-3

D-1
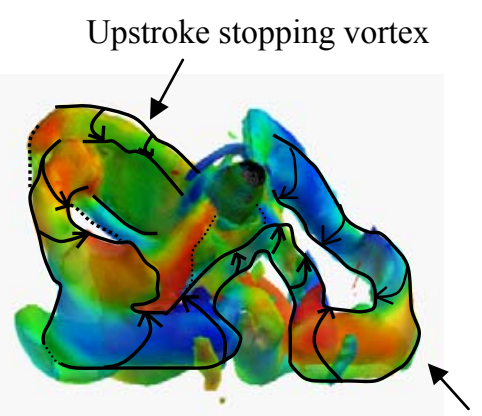

Doughnut-shaped vortex ring

D-2

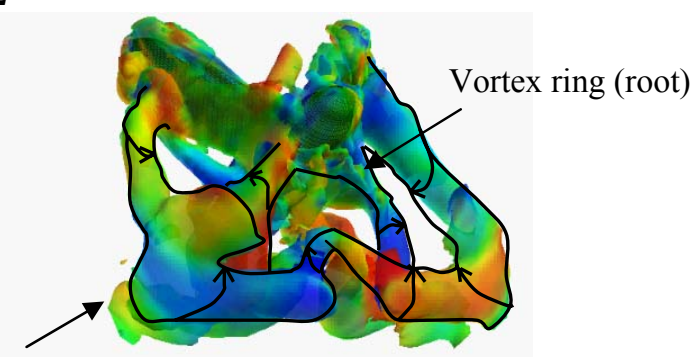




\section{D-3}

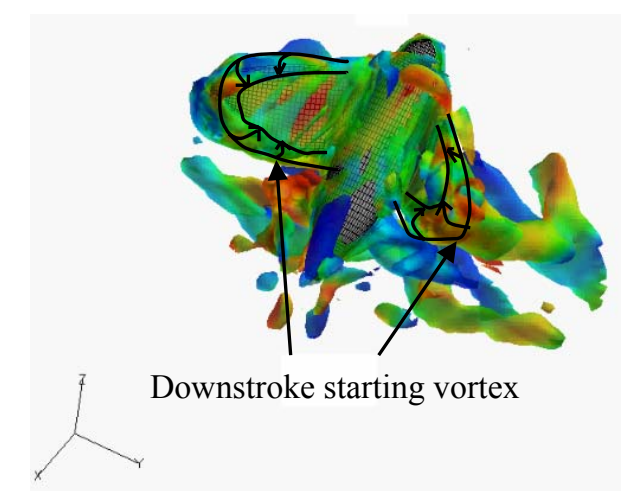

Figure 4 Visualization of flow fields around a hovering hawkmoth.

Absolute iso-vorticity surfaces around a hovering hawkmoth during (A) the downstroke, (B) the supination, (C) the upstroke, and (D) the pronation, respectively. The color of iso-vorticity surfaces indicates the normalized helicity density which defines as the projection of a fluid's spin vector in the direction of its momentum vector, being positive (red) if it points in the same direction and negative (blue) if it points in the opposite direction. 


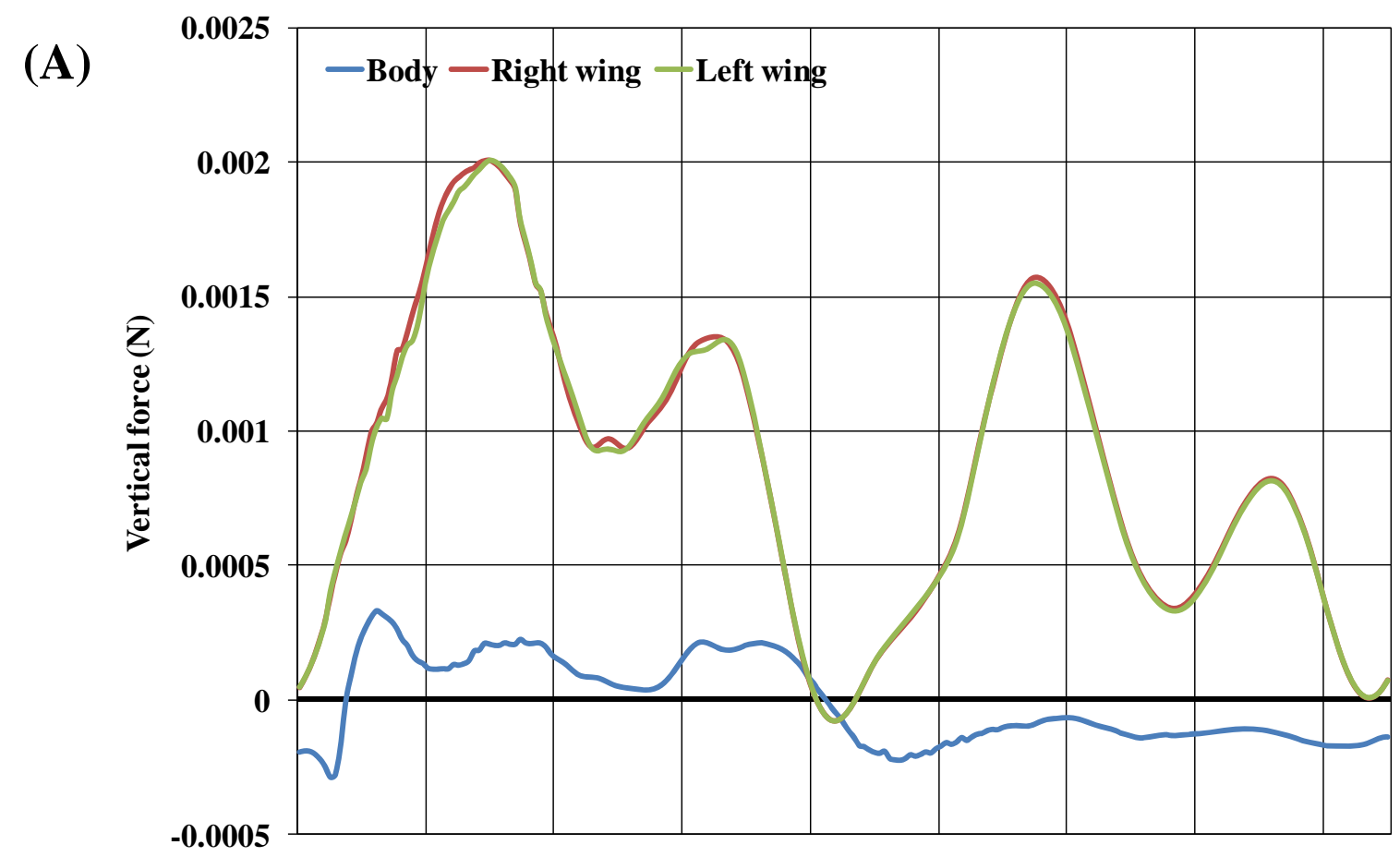

(B)

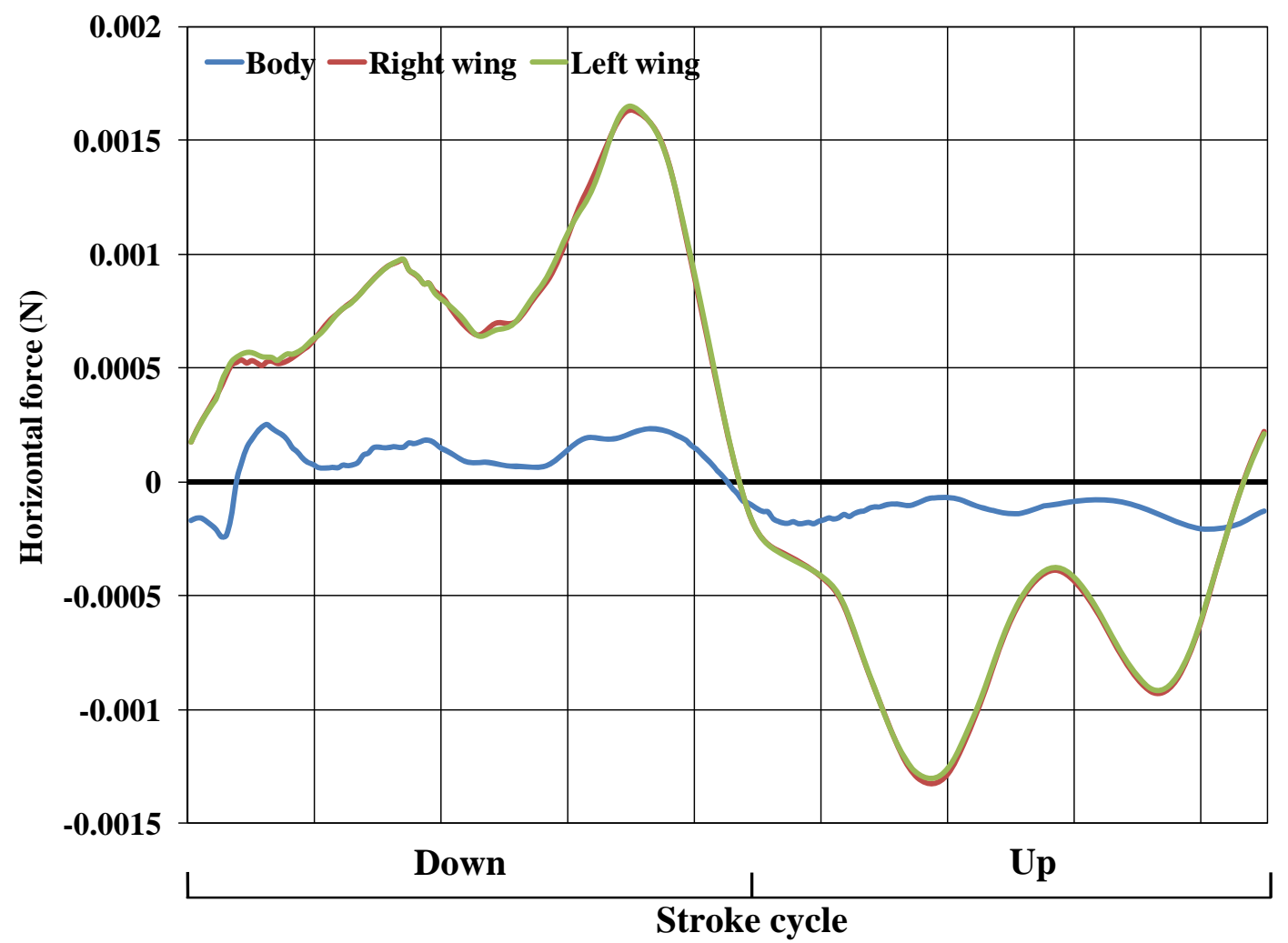


(C)

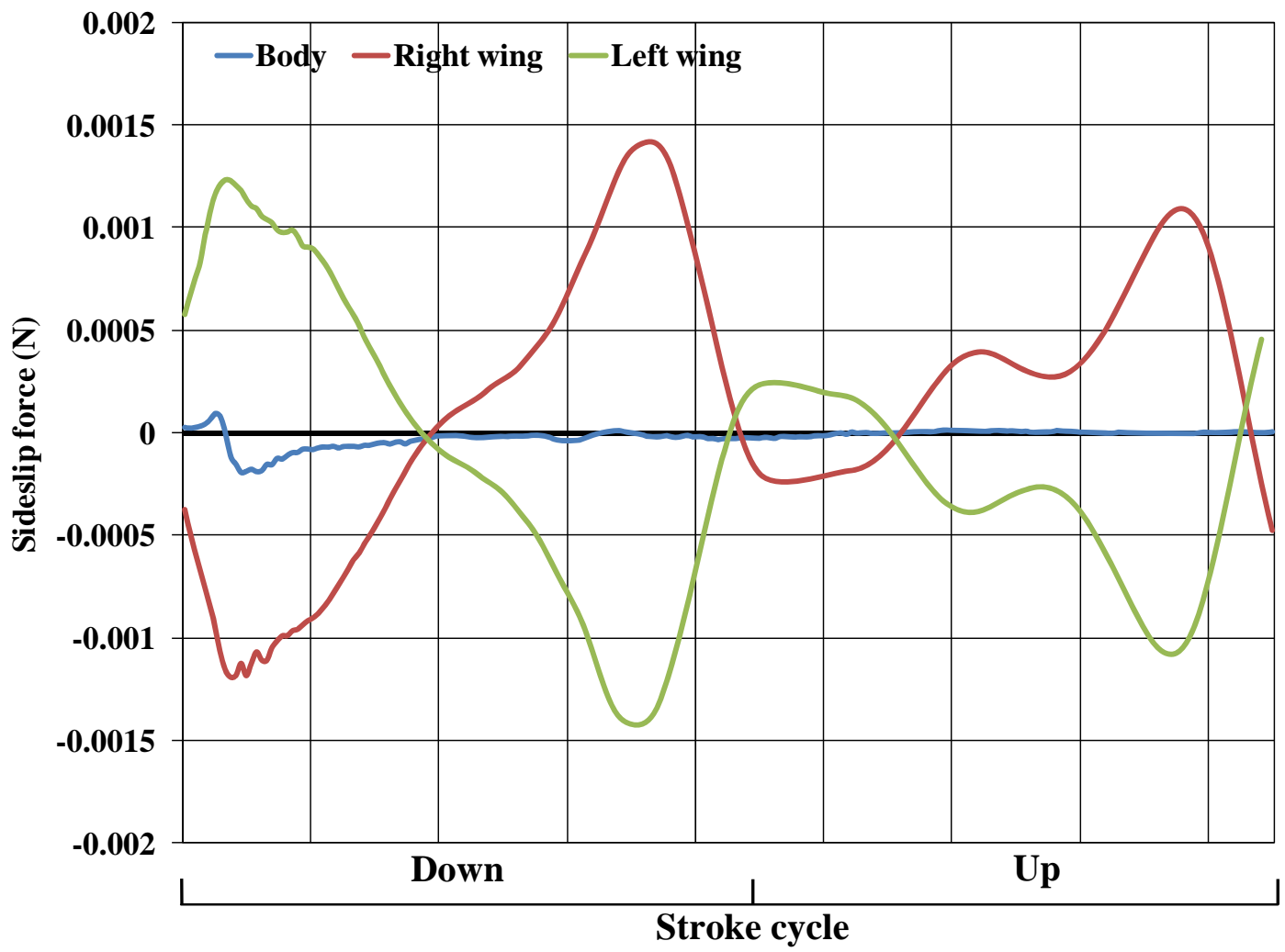

Figure 5 Time courses of aerodynamic forces over a flapping cycle.

(A) Vertical force (lift). (B) Horizontal force (drag and thrust). (C) Sideslip force. Red, green and blue lines represent aerodynamic forces acting on right wing, left wing and body, respectively. 
(A)

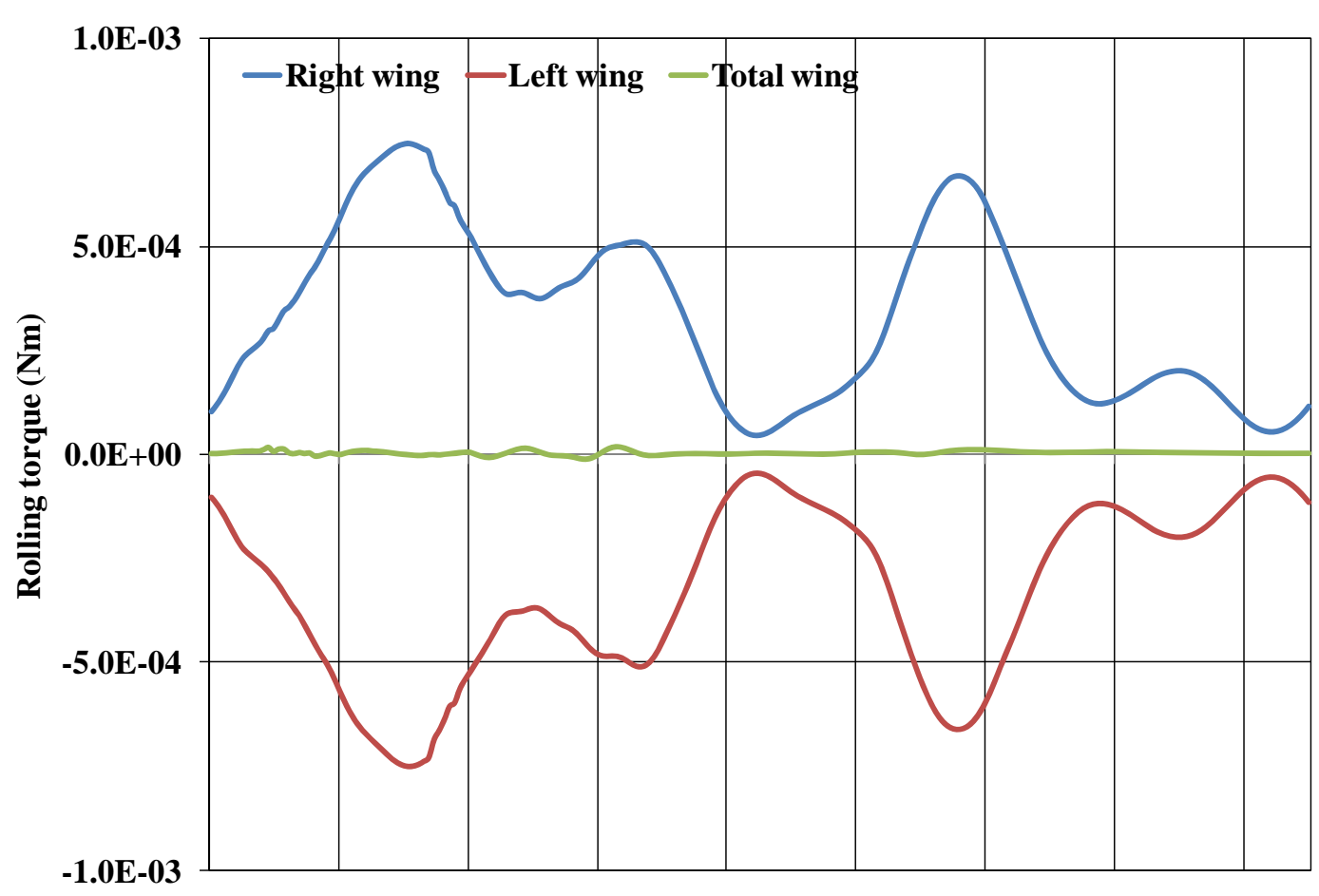

(B)

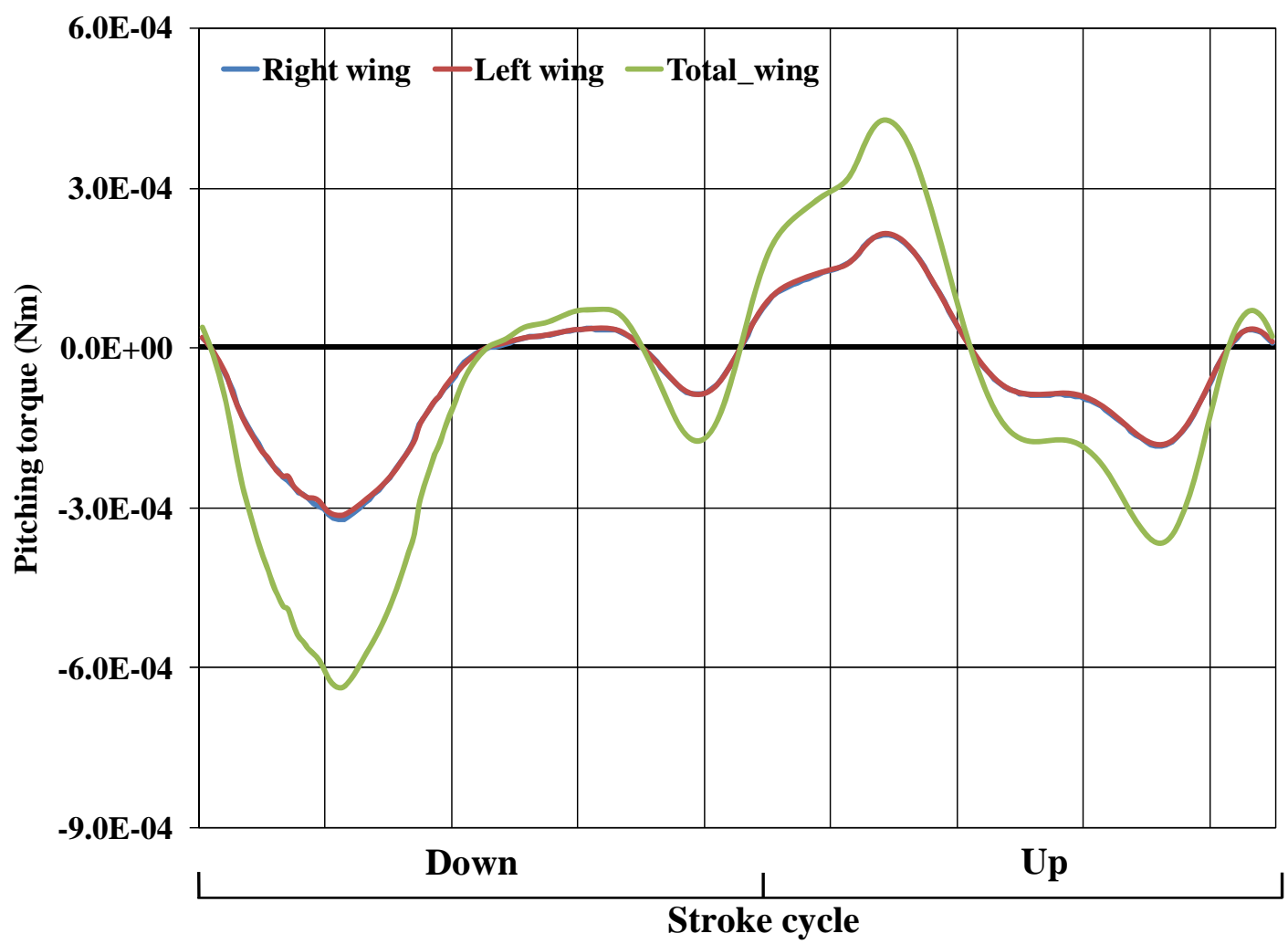


(C)

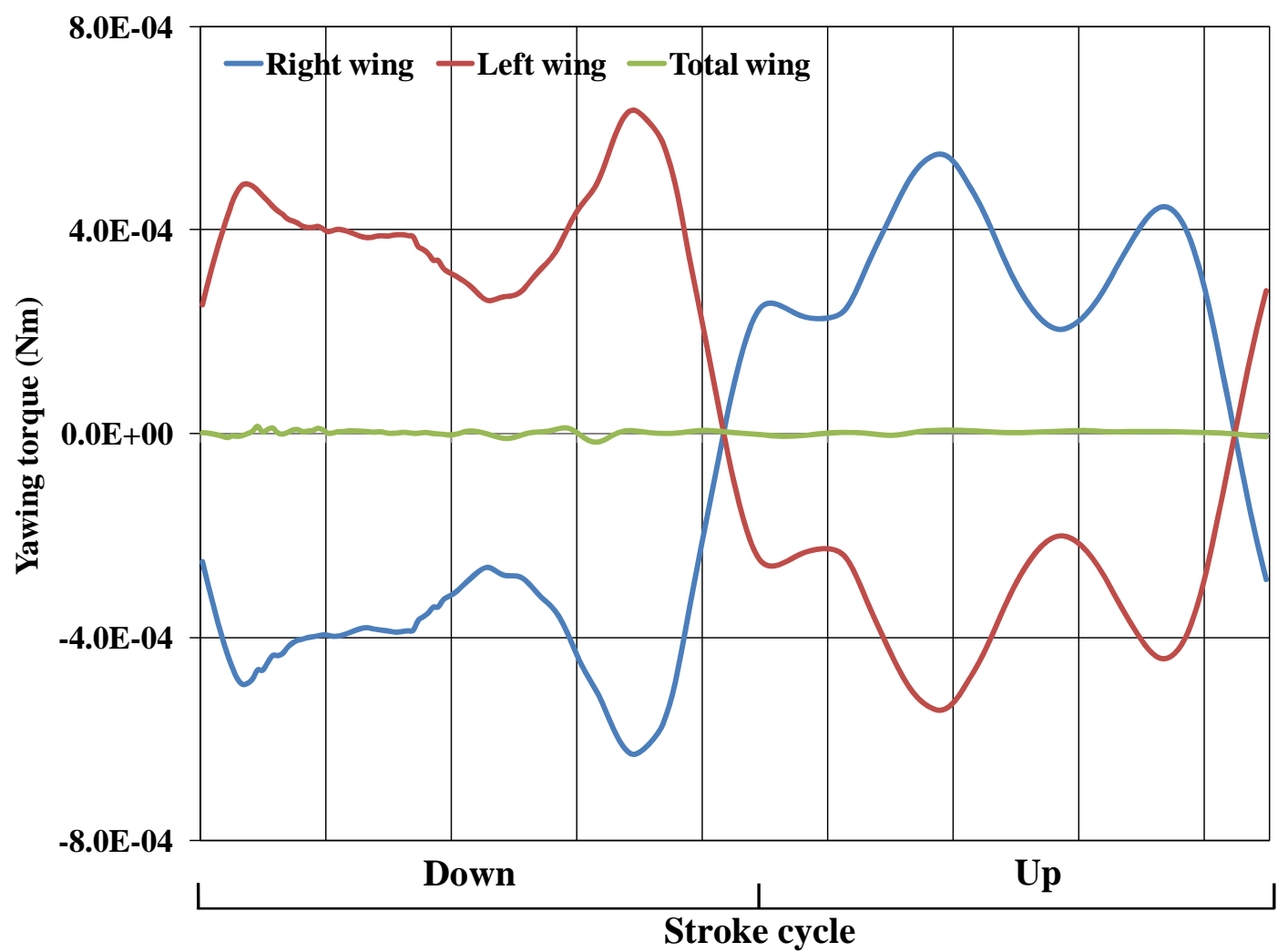

Figure 6 Time courses of aerodynamic torques of wings over a flapping cycle.

(A) Rolling torque. (B) Pitching torque. (C) Yawing torque. Red, green and blue lines represent aerodynamic torques acting on right wing, left wing and two wings ('total wing'), respectively. 


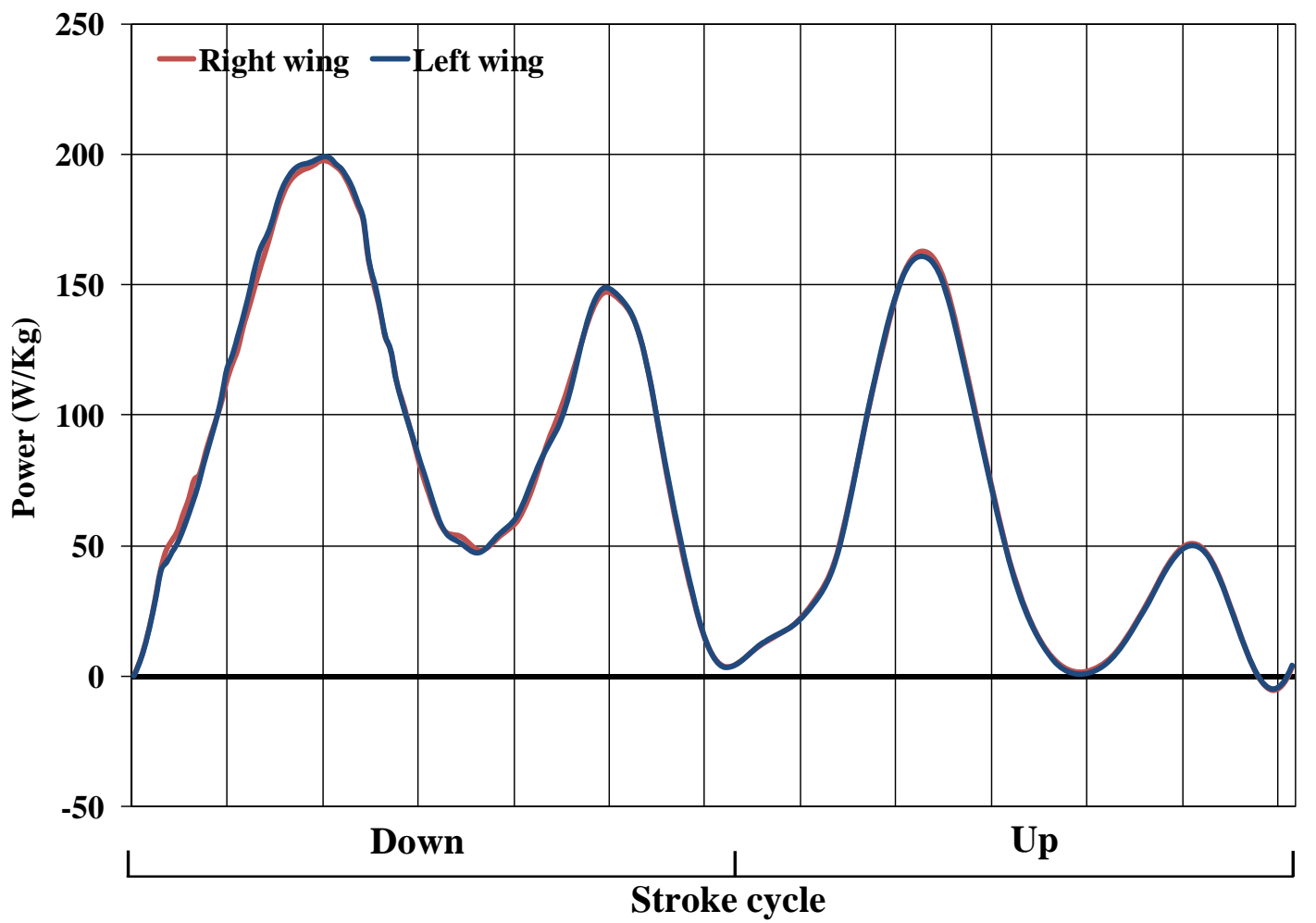

Figure 7 Time courses of the muscle-mass-specific aerodynamic power of wings over a flapping cycle.

The red and blue lines represent the muscle-mass-specific aerodynamic power are produced by the right wing and by the left wing, respectively. 